\title{
The Gray Monoidal Product of Double Categories
}

\author{
Gabriella Böhm ${ }^{1}$
}

Received: 30 January 2019 / Accepted: 18 November 2019 / Published online: 18 December 2019 (c) The Author(s) 2019

\begin{abstract}
The category of double categories and double functors is equipped with a symmetric closed monoidal structure. For any double category $\mathbb{A}$, the corresponding internal hom functor $\llbracket \mathbb{A},-\rrbracket$ sends a double category $\mathbb{B}$ to the double category whose 0 -cells are the double functors $\mathbb{A} \rightarrow \mathbb{B}$, whose horizontal and vertical 1-cells are the horizontal and vertical pseudo transformations, respectively, and whose 2-cells are the modifications. Some well-known functors of practical significance are checked to be compatible with this monoidal structure.
\end{abstract}

Keywords Double category · Gray monoidal product · Symmetric closed monoidal category

\section{Introduction}

The category 2-Cat of 2-categories and 2-functors carries different monoidal structures. The simplest one is given by the Cartesian product $\times$. It is symmetric and closed. For any 2 -category $\mathcal{A}$, the internal hom functor $\langle\mathcal{A},-\rangle$ sends a 2-category $\mathcal{B}$ to the 2-category of 2-functors $\mathcal{A} \rightarrow \mathcal{B}$, 2-natural transformations, and modifications. This is, however, often too restrictive. For example, important examples of 2-categories which are intuitively monoidal, fail to be monoids for that $[3,20,22]$. A well established generalization is the so-called Gray monoidal product $\otimes$ of [19]. It is also symmetric and closed and for any 2-category $\mathcal{A}$ the corresponding internal hom functor $[\mathcal{A},-]$ sends a 2-category $\mathcal{B}$ to the 2-category of 2functors $\mathcal{A} \rightarrow \mathcal{B}$, pseudo natural transformations, and modifications. For all 2-categories $\mathcal{A}$ and $\mathcal{B}$, the evident inclusions $\langle\mathcal{A}, \mathcal{B}\rangle \longmapsto[\mathcal{A}, \mathcal{B}]$ are the components of a natural transformation $\langle\mathcal{A},-\rangle \longmapsto[\mathcal{A},-]$. Its mate under the adjunctions $\mathcal{A} \times-\dashv\langle\mathcal{A},-\rangle$ and $\mathcal{A} \otimes-\dashv[\mathcal{A},-]$ is a natural transformation $\mathcal{A} \otimes-\rightarrow \mathcal{A} \times-$ whose components can be seen as the binary part of a monoidal structure on the identity functor $(2-C a t, \times) \rightarrow(2-C a t, \otimes)$ (but not in the opposite direction). In this sense the Cartesian monoidal structure is more restrictive than the Gray one.

The category DblCat of double categories and double functors is also symmetric closed monoidal via the Cartesian product $\times$. For any double category $\mathbb{A}$, the corresponding internal

Communicated by Nicola Gambino.

Gabriella Böhm

bohm.gabriella@wigner.mta.hu

1 Wigner Research Centre for Physics, P.O.B. 49, Budapest 114 1525, Hungary 
hom functor $\langle\mathbb{A},-\rangle$ sends a double category $\mathbb{B}$ to the double category whose 0 -cells are the double functors $\mathbb{A} \rightarrow \mathbb{B}$, whose horizontal and vertical 1-cells are the horizontal and vertical transformations, respectively, and whose 2-cells are the modifications; see [15]. The analogue of the Gray monoidal product on DblCat, however, has apparently not yet been discussed in the literature. The current paper addresses this question.

For any double categories $\mathbb{A}$ and $\mathbb{B}$, there is a bigger double category $\llbracket \mathbb{A}, \mathbb{B} \rrbracket$ in which the 0 -cells are still the double functors $\mathbb{A} \rightarrow \mathbb{B}$. The horizontal and vertical 1-cells are, however, the horizontal and vertical pseudo (or strong) transformations of [15]. The 2-cells are their modifications. In Sect. 2 we use [6, Lemma 3.9] to prove that for any double categories $\mathbb{A}$ and $\mathbb{B}$, there is a representing object $\mathbb{B} \otimes \mathbb{A}$ of the functor DblCat $(\mathbb{B}, \llbracket \mathbb{A},-\rrbracket):$ DblCat $\rightarrow$ Set. Constructing the associativity and unit constraints, as well as the symmetry, in Sect. 3 we show that $\otimes$ equips DblCat with a symmetric monoidal structure. (We use the term monoidal category in the same sense as in Sections VII.1 and XI.1 of [21]; that is, associativity and unitality of the monoidal product are required only up-to coherent natural isomorphisms. For the notion of symmetry we refer to Section XI.1 of [21].) In order to support this choice of monoidal structure on DblCat, in Sect. 4 monoidality of the following functors is checked.

- The identity functor (DblCat, $\times) \rightarrow($ DblCat, $\otimes)$.

- The functors (DblCat, $\otimes) \rightarrow(2-C a t, \otimes)$ sending double categories to their horizontalor vertical-2-categories (for the Gray monoidal product $\otimes$ on 2-Cat).

- The square (or quintet) construction functor $\mathbb{S q r}:(2-C a t, \otimes) \rightarrow($ DblCat, $\otimes)$ due to Ehresmann [9].

- The functor Mnd : (DblCat, $\otimes) \rightarrow($ DblCat, $\otimes)$, sending a double category to the double category of its monads by Fiore, Gambino and Kock [10].

(Again, the term monoidal functor is used in the same lax sense as in Section XI.2 of [21]. That is, a monoidal functor consists of coherent natural transformations which control the difference between the monoidal structure of the target category, and the image of the monoidal structure of the source category.)

We also give an explicit description of monoids in (DblCat, $\otimes$ ) which generalize the strict monoidal double categories of [7]; that is, the monoids in (DblCat, $\times$ ). They turn out to give rise to monoids in the category of double categories and double pseudo functors in the sense of [23, Definition 6.1], with respect to the Cartesian product. They do not seem to allow, however, for an interpretation as monoidal double categories in the sense of [24, Definition 2.9], [18, Section 3.1], [12, Definition 31] and [16, Section 5.5]; orwhat is the same-as one object locally cubical bicategories in [13]. That is to say, they do not seem to give rise to pseudo monoids in the monoidal bicategory of double categories, their homomorphisms in $[12,16,18,24]$ and say, vertical transformations with the Cartesian product.

The author's personal motivation to study the Gray monoidal product of double categories arose from the work on [4]. In that paper, lifting of so-called multimonoidal structures (in the sense of [1]) to Eilenberg-Moore objects of multimonoidal monads is described in terms of a strict monoidal double functor between strict monoidal double categories (that is, monoids in DblCat with respect to the Cartesian product). While this is applicable to multimonoidal monads on nice enough categories as in [2], a generalization from Cat to more interesting Gray monoids (that is, monoids in 2-Cat with respect to the Gray tensor product) requires a notion of monoids in DblCat with respect to a suitable Gray monoidal product $\otimes$.

There should be further interesting applications.

Gray categories - that is, categories enriched in (2-Cat, $\otimes)$ - solve the coherence problem of tricategories: in [14] any tricategory is proven to be triequivalent to a Gray category. The 
Gray monoidal product $\otimes$ of DblCat is hoped to play some role in the coherence of triple categories.

One object groupoidal Gray categories are algebraic models of homotopy 3-types; an interesting application would be the appearance of (DblCat, $\otimes)$ in some homotopy types.

2-Cat with the Gray tensor product is a monoidal model category. One can ask if the model structures on the category of small double categories in [11] are monoidal with respect to the Gray monoidal product in the current paper.

\section{Existence}

In this section we construct an adjunction $-\otimes \mathbb{D} \dashv \llbracket \mathbb{D},-\rrbracket$ of endofunctors on the category DblCat of double categories, for any double category $\mathbb{D}$. Our line of reasoning is similar to [6, Proposition 3.10]. The occurring double functor $\otimes:$ DblCat $\times$ DblCat $\rightarrow$ DblCat is our candidate Gray monoidal product on DblCat. Mac Lane's coherence conditions are checked in Sect. 3.

\subsection{The Category of Double Categories}

We begin with introducing the category DblCat of double categories and double functors, and recording some of its basic properties.

Definition 2.1 A double category is an internal category in the category Cat of categories and functors. A double functor in an internal functor in Cat. Double categories are the objects, and double functors are the morphisms of the category DblCat.

So a double category consists of 0 -cells, also called objects, (interpreted as the objects of the category of objects), vertical 1-cells (which are the morphisms of the category of objects), horizontal 1-cells (the objects of the category of morphisms) and 2-cells (the morphisms of the category of morphisms). They can be composed vertically (in the category of objects and the category of morphisms, respectively) and horizontally (via the composition functor of the double category). As usual in the literature (see e.g. [15]), we denote 2-cells as squares surrounded by the appropriate horizontal and vertical source and target 1-cells. We denote by 1 both horizontal and vertical identity 1-cells; and also identity 2 -cells for the horizontal or vertical composition. Usually we neither make notational difference between the compositions of horizontal and vertical 1-cells; both are denoted by a dot (if not a diagram is rather drawn).

By [11, Theorem 4.1] and its proof, DblCat is locally finitely presentable—so in particular cocomplete-and complete. Its terminal object $\mathbb{1}$ is the double category of a single object and only identity higher cells.

Consider the double category $\mathbb{G}$ which is freely generated by a single 2-cell. In more detail, $\mathbb{G}$ has four objects, we denote them by $X, Y, V$ and $Z$. There are identity horizontal and vertical identity 1-cells for each object as well as non-identity horizontal and vertical 1 -cells

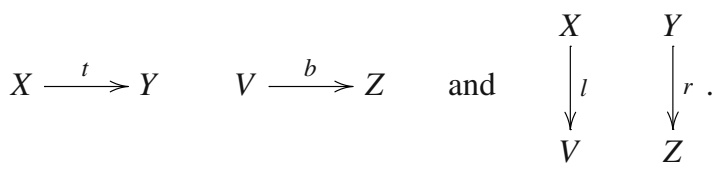


There are vertical identity 2-cells at each horizontal 1-cell, horizontal identity 2-cells at each vertical 1-cell, and a single non-identity 2 -cell

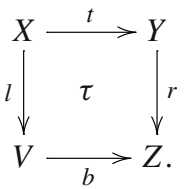

The functor DblCat $(\mathbb{G},-):$ DblCat $\rightarrow$ Set sends a double category $\mathbb{A}$ to the set of double functors $\mathbb{G} \rightarrow \mathbb{A}$, which can be identified with the set of 2-cells in $\mathbb{A}$. A double functor $F$ is sent to its 2-cell part, which is an isomorphism in Set if and only if $\mathrm{F}$ is bijective on the 2-cells. Since this includes bijectivity also on the identity 2-cells of various kinds, it is equivalent to $\mathrm{F}$ being bijective on all kinds of cells; that is, its being an isomorphism in DblCat. By the so obtained conservativity of the functor $\operatorname{DblCat}(\mathbb{G},-):$ DblCat $\rightarrow$ Set we conclude that $\mathbb{G}$ is a strong generator of the finitely complete category DblCat with coproducts, see [5, Proposition 4.5.10].

\subsection{The Double Categories of Double Functors}

Using similar constructions to those in [15, Section 7], any double categories $\mathbb{A}$ and $\mathbb{B}$ determine a double category $\llbracket \mathbb{A}, \mathbb{B} \rrbracket$ as follows.

The 0 -cells are the double functors $\mathbb{A} \rightarrow \mathbb{B}$.

The horizontal 1-cells are the horizontal pseudo transformations (called strong horizontal transformations in [15, Section 7.4]). A horizontal pseudo transformation $x: \mathrm{F} \rightarrow \mathrm{G}$ consists of the following data.

- For any 0-cell $A$ of $\mathbb{A}$, a horizontal morphism in $\mathbb{B}$ on the left;

- for any vertical 1-cell $f$ in $\mathbb{A}$, a 2-cell in $\mathbb{B}$ in the middle;

- for any horizontal 1-cell $h$ in $\mathbb{A}$, a vertically invertible 2-cell in $\mathbb{B}$ on the right:
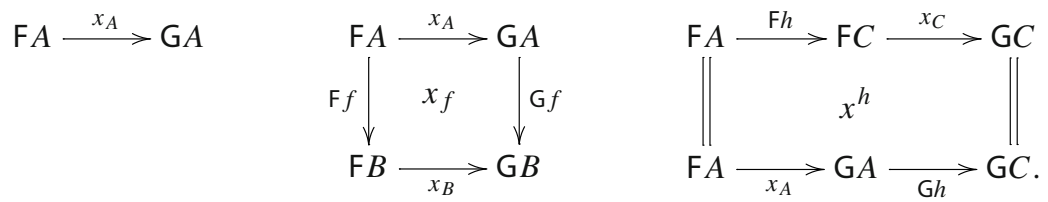

These ingredients are subject to the following axioms.

(i) Vertical functoriality, saying that for the identity vertical 1-cell 1 on any object $A$ in $\mathbb{A}, x_{1}$ is equal to the vertical identity 2 -cell on the left; and for any composable vertical 1-cells $f$ and $g$ in $\mathbb{A}$, the equality on the right holds:
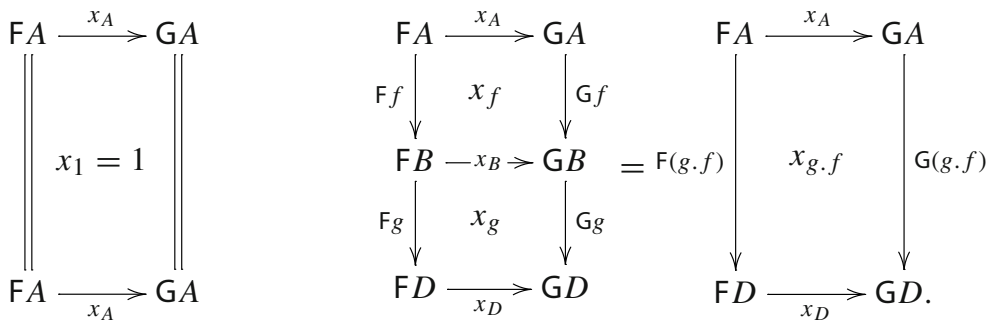
(ii) Horizontal functoriality, saying that for the identity horizontal 1-cell 1 on any object $A, x^{1}$ is equal to the same vertical identity 2 -cell on the left; and for any composable horizontal 1-cells $h$ and $k$ in $\mathbb{A}$, the equality on the right holds:
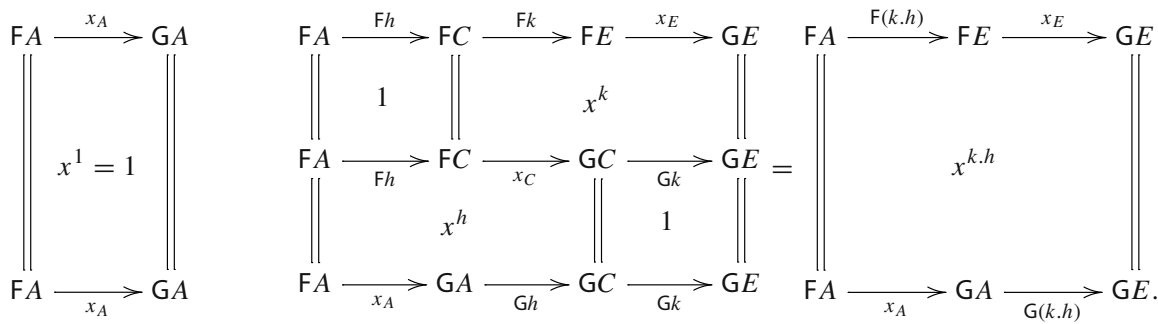

(iii) Naturality, saying that for any 2-cell $\omega$ in $\mathbb{A}$,

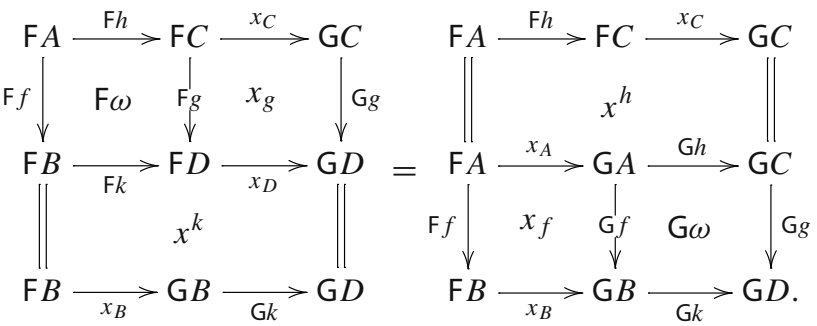

The vertical 1-cells are the vertical pseudo transformations (called strong vertical transformations in [15, Section 7.4]). A vertical pseudo transformation $y: \mathrm{F} \rightarrow \mathrm{H}$ consists of the following data.

- For any 0 -cell $A$ of $\mathbb{A}$, a vertical 1 -cell in $\mathbb{B}$ on the left;

- for any horizontal 1-cell $h$ in $\mathbb{A}$, a 2-cell in $\mathbb{B}$ in the middle;

- for any vertical 1 -cell $f$ in $\mathbb{A}$, a horizontally invertible 2-cell in $\mathbb{B}$ on the right:
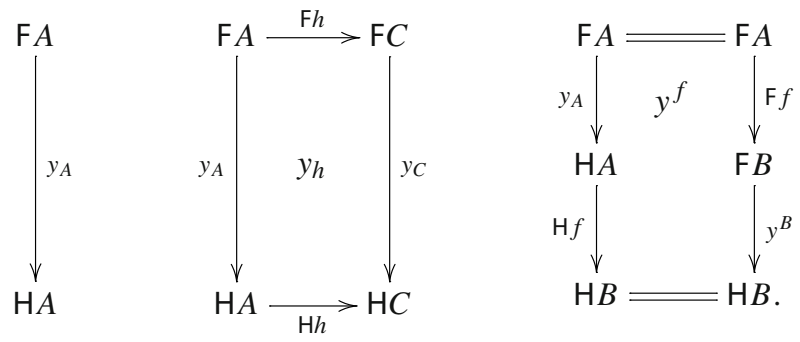

These ingredients are subject to the following axioms.

(i) Horizontal functoriality, saying that for the identity horizontal 1-cell 1 on any object $A$ in $\mathbb{A}, y_{1}$ is equal to the horizontal identity 2-cell on the left; and for any composable horizontal 1-cells $h$ and $k$ in $\mathbb{A}$, the equality on the right holds:
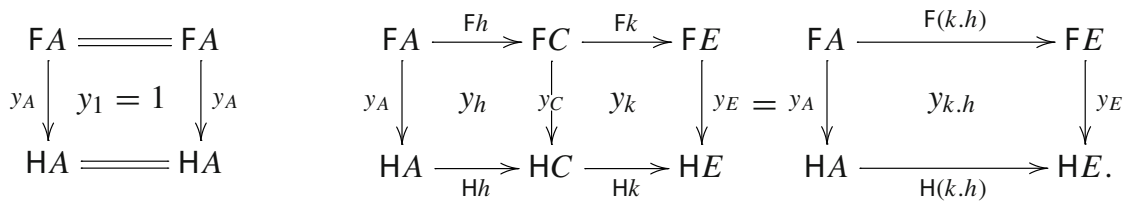
(ii) Vertical functoriality, saying that for the identity vertical 1-cell 1 on any object $A, y^{1}$ is equal to the same horizontal identity 2-cell on the left; and for any composable vertical 1 -cells $f$ and $g$ in $\mathbb{A}$, the equality on the right holds:
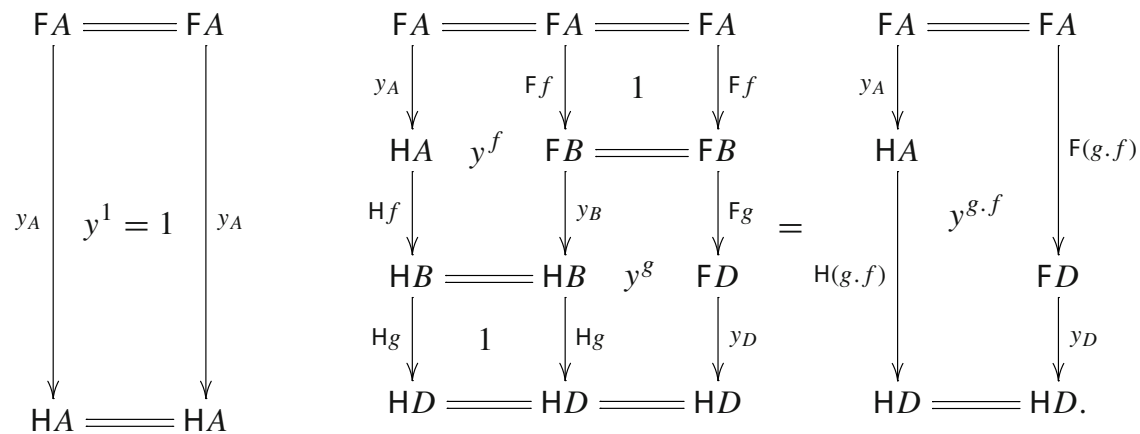

(iii) Naturality, saying that for any 2-cell $\omega$ in $\mathbb{A}$,

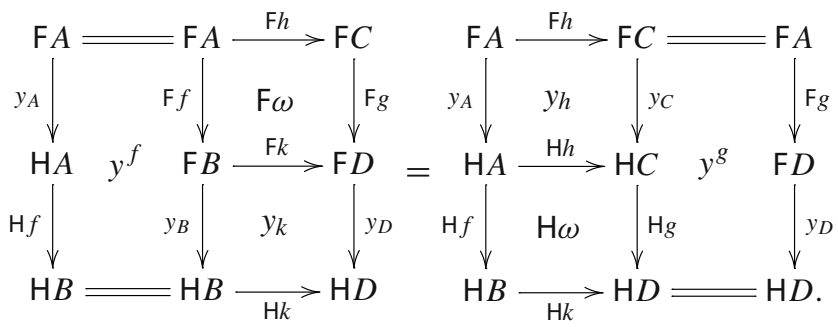

The 2-cells are the modifications. A modification on the left is given by a collection of 2-cells in $\mathbb{B}$ on the right, for all 0-cells $A$ of $\mathbb{A}$ :
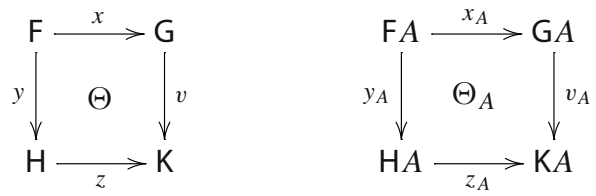

satisfying the following axioms.

(i) For any horizontal 1-cell $h$ in $\mathbb{A}$,

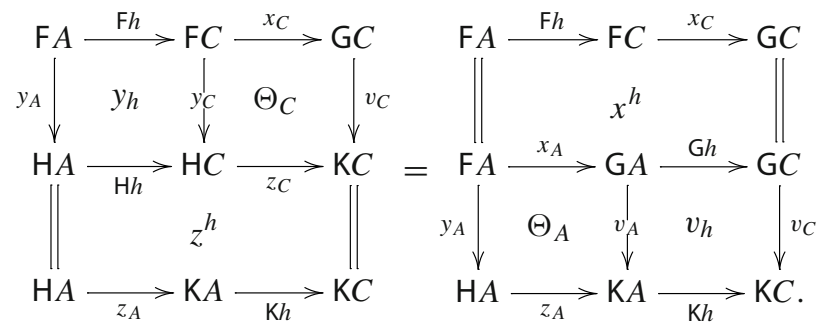


(ii) For any vertical 1 -cell $f$ in $\mathbb{A}$,

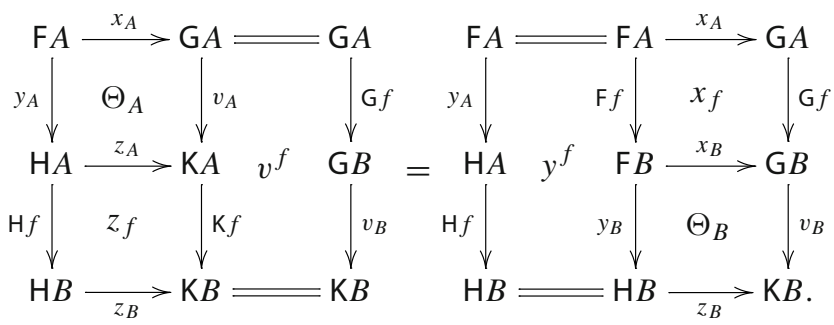

The identity horizontal pseudo transformation has the components
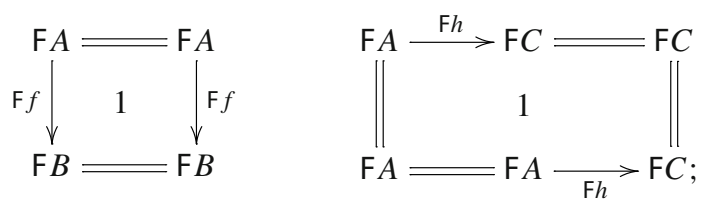

while the composite of some horizontal pseudo transformations $\mathrm{F} \stackrel{x}{\rightarrow} \mathrm{G} \stackrel{z}{\rightarrow} \mathrm{H}$ has the components
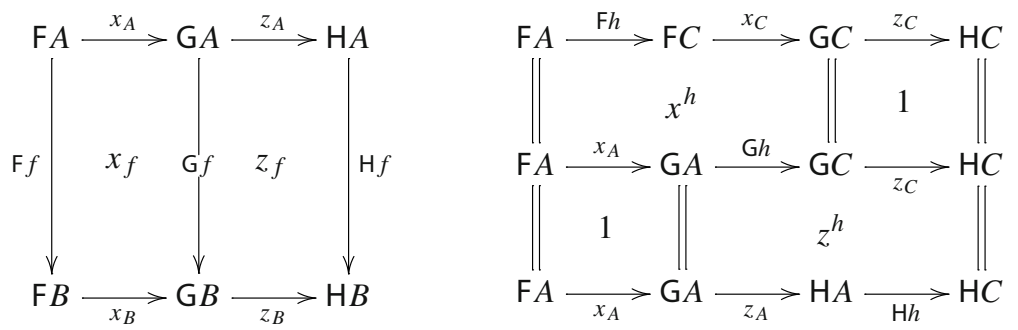

for any horizontal 1-cell $h$ and vertical 1-cell $f$ in $\mathbb{A}$. Symmetrically, the identity vertical pseudo transformation has the components
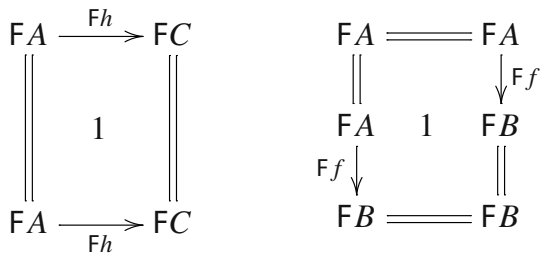
and the composite of vertical pseudo transformations $\mathrm{F} \stackrel{y}{\longrightarrow} \mathrm{G} \stackrel{v}{\longrightarrow} \mathrm{H}$ has the components
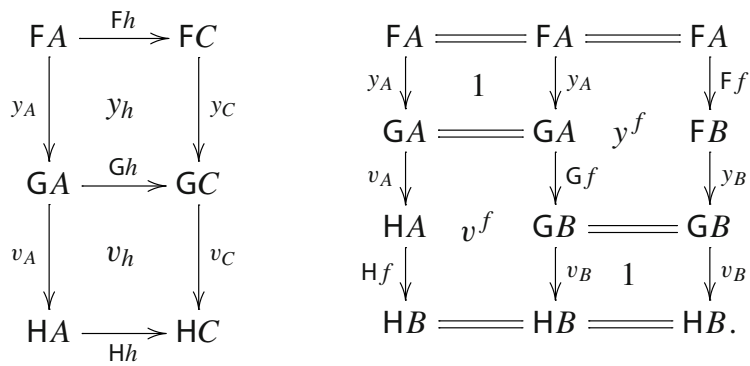

The components of the horizontal composite of modifications on the left of
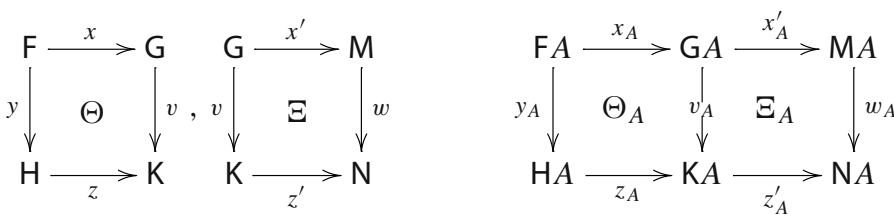

are the horizontal composites of their components on the right. Symmetrically, the components of the vertical composite of modifications on the left of
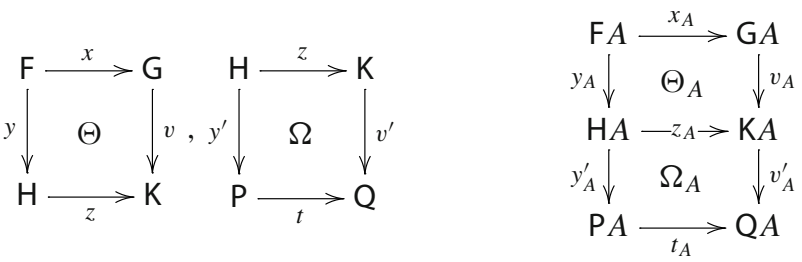

are the vertical composites of their components on the right.

Throughout, we identify any double category $\mathbb{A}$ with the isomorphic double category $\llbracket \mathbb{1}, \mathbb{A} \rrbracket$.

\subsection{The Functor $\llbracket-,-\rrbracket:$ DblCat $^{\text {op }} \times$ DblCat $\rightarrow$ DblCat}

In this section we interpret the map, sending a pair of double categories $\mathbb{A}$ and $\mathbb{B}$ to the double category $\llbracket \mathbb{A}, \mathbb{B} \rrbracket$ of Sect. 2.2 , as the object map of a functor in the title. So we need to construct its morphism map, sending a pair of double functors $F: \mathbb{A}^{\prime} \rightarrow \mathbb{A}$ and $G: \mathbb{B} \rightarrow \mathbb{B}^{\prime}$ to a double functor $\llbracket \mathrm{F}, \mathrm{G} \rrbracket: \llbracket \mathbb{A}, \mathbb{B} \rrbracket \rightarrow \llbracket \mathbb{A}^{\prime}, \mathbb{B}^{\prime} \rrbracket$.

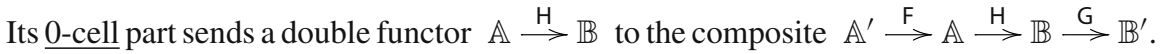

The horizontal 1-cell part sends a horizontal pseudo transformation $\mathrm{H} \stackrel{x}{\rightarrow} \mathrm{H}^{\prime}$ to the horizontal pseudo transformation with the components
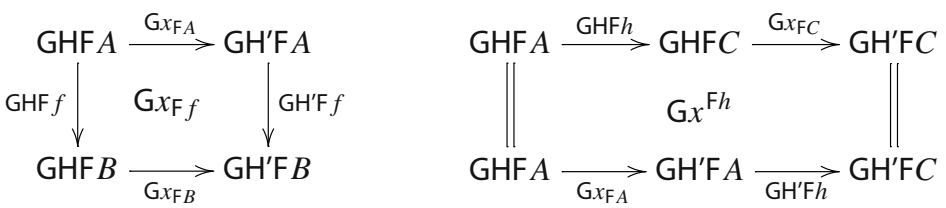
for any horizontal 1-cell $h$ and vertical 1-cell $f$ in $\mathbb{A}^{\prime}$.

Symmetrically, the vertical 1-cell part sends a vertical pseudo transformation $y$ to the vertical pseudo transformation with the components
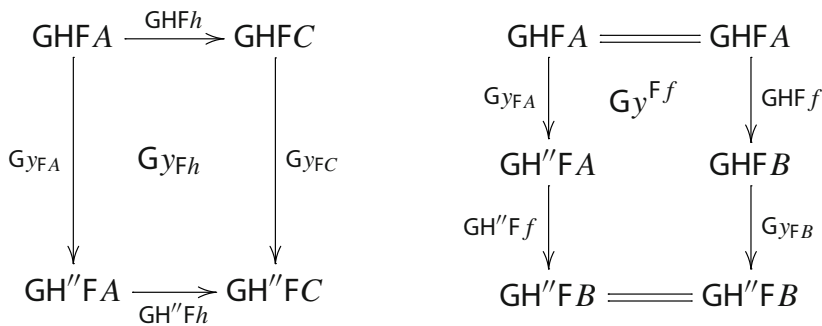

for any horizontal 1-cell $h$ and vertical 1-cell $f$ in $\mathbb{A}^{\prime}$.

Finally, the 2-cell part sends a modification in the first diagram to the modification with components in the second diagram:
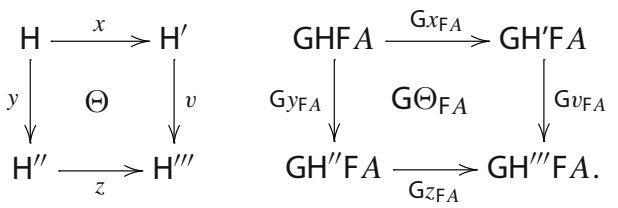

\subsection{The Extranatural Transformation $\mathfrak{l}$}

In this section we construct an extranatural transformation

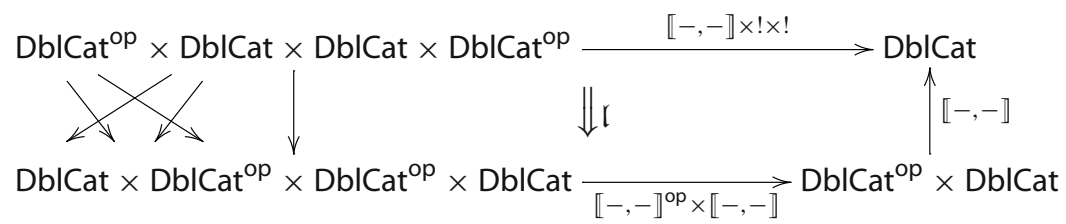

where! in the top row denotes the unique functor to the terminal category, and in the left column the depicted symmetry natural isomorphism - that is, the appropriate flip map sending $(\mathbb{A}, \mathbb{B}, \mathbb{C}, \mathbb{D})$ to $(\mathbb{C}, \mathbb{A}, \mathbb{D}, \mathbb{B})$-occurs. We denote by lower indices that $l$ is ordinary natural in the first two arguments, and an upper index reminds us that it is extranatural in the last two factors. At any object of the form $\mathbb{A}, \mathbb{B}, \mathbb{D}, \mathbb{D}$, it is given by the following double functor $\mathfrak{l}_{\mathbb{A}, \mathbb{B}}^{\mathbb{D}}: \llbracket \mathbb{A}, \mathbb{B} \rrbracket \rightarrow \llbracket \llbracket \mathbb{D}, \mathbb{A} \rrbracket, \llbracket \mathbb{D}, \mathbb{B} \rrbracket \rrbracket$.

The 0 -cell part sends a double functor $G: \mathbb{A} \rightarrow \mathbb{B}$ to the double functor $\llbracket 1, G \rrbracket: \llbracket \mathbb{D}, \mathbb{A} \rrbracket \rightarrow$ $\llbracket \mathbb{D}, \mathbb{B} \rrbracket$ of Sect. 2.3.

The horizontal 1-cell part sends a horizontal pseudo transformation $G \stackrel{x}{\rightarrow} G^{\prime}$ to the horizontal pseudo transformation $\llbracket 1, G \rrbracket \rightarrow \llbracket 1, G^{\prime} \rrbracket$ with the following components, for any horizontal pseudo transformation $p$ and vertical pseudo transformation $q$ between double functors $\mathrm{H}, \mathrm{H}^{\prime}: \mathbb{D} \rightarrow \mathbb{A}$. 
- The horizontal pseudo transformation with components
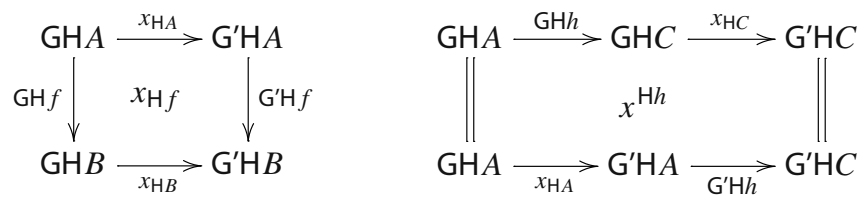

for any horizontal 1-cell $h$ and vertical 1-cell $f$ in $\mathbb{D}$.

- The modification with components

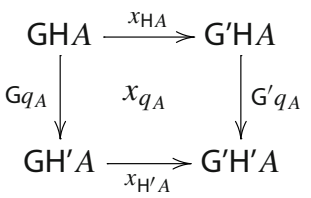

for any 0 -cell $A$ in $\mathbb{D}$.

- The vertically invertible modification with components

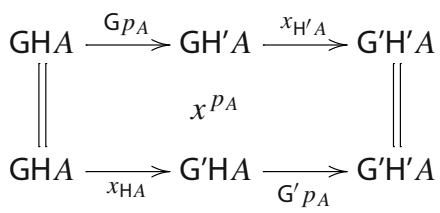

for any 0 -cell $A$ in $\mathbb{D}$.

Symmetrically, the vertical 1-cell part sends a vertical pseudo transformation $y$ from $G$ to $\mathrm{G}^{\prime}$ to the vertical pseudo transformation from $\llbracket 1, \mathrm{G} \rrbracket$ to $\llbracket 1, \mathrm{G}^{\prime} \rrbracket$ with the following components, for any horizontal pseudo transformation $p$ and vertical pseudo transformation $q$ between double functors $\mathrm{H}, \mathrm{H}^{\prime}: \mathbb{D} \rightarrow \mathbb{A}$.

- The vertical pseudo transformation with components
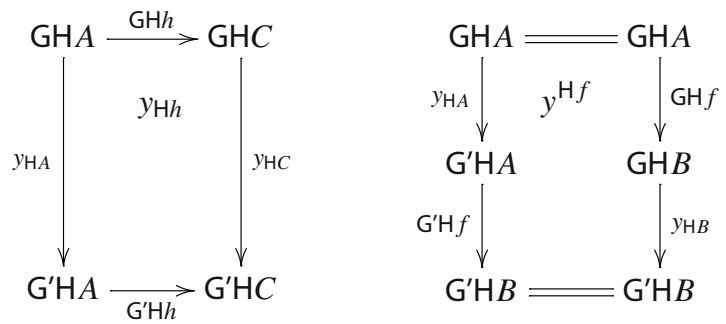

for any horizontal 1-cell $h$ and vertical 1 -cell $f$ in $\mathbb{D}$.

- The modification with components

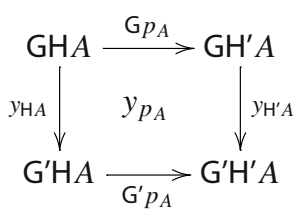

for any 0 -cell $A$ in $\mathbb{D}$. 
- The horizontally invertible modification with components

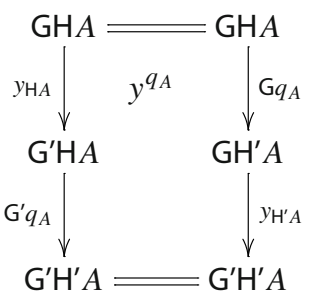

for any 0 -cell $A$ in $\mathbb{D}$.

The 2 -cell part sends a modification in the first diagram to the modification with components in the second diagram, for any 0 -cell $A$ in $\mathbb{D}$ :
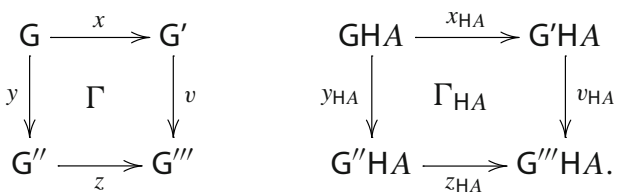

For any double categories $\mathbb{A}, \mathbb{B}, \mathbb{C}$ and $\mathbb{D}$, direct computation verifies the commutativity of

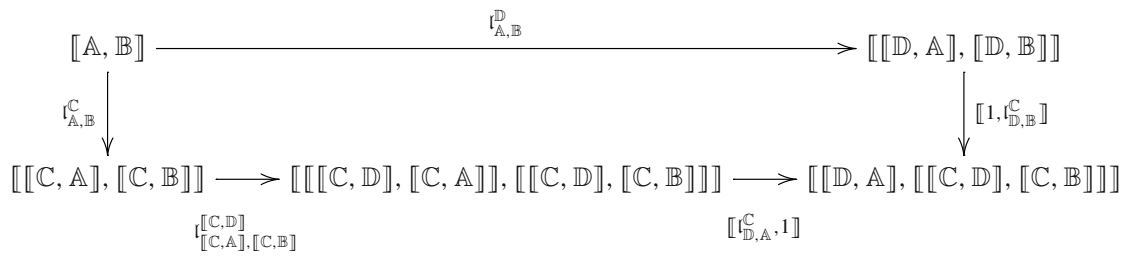

and the equality of

$$
\llbracket \mathbb{A}, \mathbb{B} \rrbracket \stackrel{\mathfrak{I}_{\mathbb{A}, \mathbb{B}}^{\mathbb{B}}}{\longrightarrow} \llbracket \llbracket \mathbb{A}, \mathbb{A} \rrbracket, \llbracket \mathbb{A}, \mathbb{B} \rrbracket \rrbracket \stackrel{\llbracket 1_{\mathbb{A}}, 1 \rrbracket}{\longrightarrow} \llbracket \mathbb{1}, \llbracket \mathbb{A}, \mathbb{B} \rrbracket \rrbracket \cong \llbracket \mathbb{A}, \mathbb{B} \rrbracket
$$

to the identity double functor, where $1_{\mathbb{A}}: \mathbb{1} \rightarrow \llbracket \mathbb{A}, \mathbb{A} \rrbracket$ is the double functor sending the single object of $\mathbb{1}$ to the identity double functor $1_{\mathbb{A}}: \mathbb{A} \rightarrow \mathbb{A}$.

\subsection{The Extranatural Transformation $\mathfrak{r}$}

In this section we construct another extranatural transformation

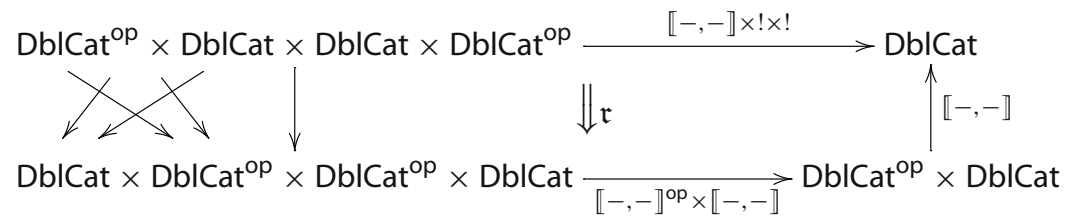

where again, ! in the top row denotes the unique functor to the terminal category, and in the left column the depicted symmetry natural isomorphism - that is, the appropriate flip map 
sending $(\mathbb{A}, \mathbb{B}, \mathbb{C}, \mathbb{D})$ to $(\mathbb{B}, \mathbb{D}, \mathbb{A}, \mathbb{C})$ - occurs. As in Sect. 2.4 , we denote by lower indices that $\mathfrak{r}$ is ordinary natural in the first two arguments, and an upper index reminds us that it is extranatural in the last two factors. At any object of the form $\mathbb{A}, \mathbb{B}, \mathbb{D}, \mathbb{D}$, it is given by the following double functor $\mathfrak{r}_{\mathbb{A}, \mathbb{B}}^{\mathbb{D}}: \llbracket \mathbb{A}, \mathbb{B} \rrbracket \rightarrow \llbracket \llbracket \mathbb{B}, \mathbb{D} \rrbracket, \llbracket \mathbb{A}, \mathbb{D} \rrbracket \rrbracket$.

The 0 -cell part sends a double functor $F: \mathbb{A} \rightarrow \mathbb{B}$ to the double functor $\llbracket F, 1 \rrbracket: \llbracket \mathbb{B}, \mathbb{D} \rrbracket \rightarrow$ $\llbracket \mathbb{A}, \mathbb{D} \rrbracket$ in Sect. 2.3.

The horizontal 1-cell part sends a horizontal pseudo transformation $\mathrm{F} \stackrel{x}{\rightarrow} \mathrm{F}^{\prime}$ to the horizontal pseudo transformation $\llbracket \mathrm{F}, 1 \rrbracket \rightarrow \llbracket \mathrm{F}^{\prime}, 1 \rrbracket$ with the following components, for any horizontal pseudo transformation $p$ and vertical pseudo transformation $q$ between double functors $\mathrm{H}, \mathrm{H}^{\prime}: \mathbb{B} \rightarrow \mathbb{D}$.

- The horizontal pseudo transformation with components
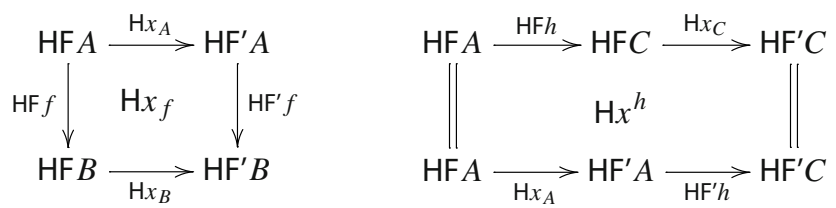

for horizontal 1-cells $h$ and vertical 1-cells $f$ in $\mathbb{A}$.

- The modification with the components

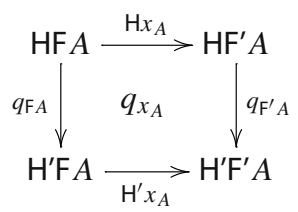

for any 0 -cell $A$ in $\mathbb{A}$.

- The vertically invertible modification with the components

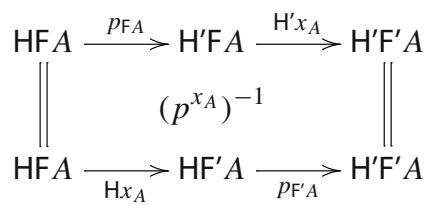

for any 0 -cell $A$ in $\mathbb{A}$.

Symmetrically, the vertical 1-cell part sends a vertical pseudo transformation $y$ from $\mathrm{F}$ to $F^{\prime}$ to the vertical pseudo transformation $\llbracket F, 1 \rrbracket$ to $\llbracket F^{\prime}, 1 \rrbracket$ with the following components, for any horizontal pseudo transformation $p$ and vertical pseudo transformation $q$ between double functors $\mathrm{H}, \mathrm{H}^{\prime}: \mathbb{B} \rightarrow \mathbb{D}$.

- The vertical pseudo transformation with components
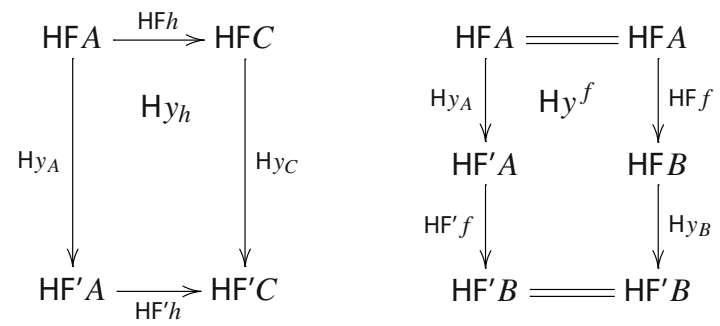
for horizontal 1-cells $h$ and vertical 1-cells $f$ in $\mathbb{A}$.

- The modification with the components

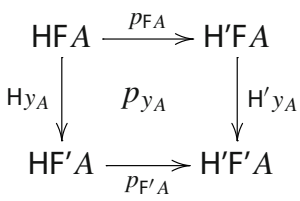

for any 0 -cell $A$ in $\mathbb{A}$.

- The horizontally invertible modification with the components

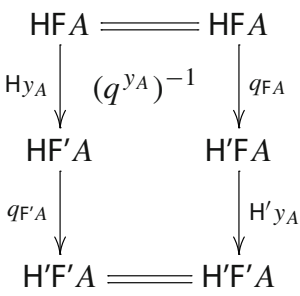

for any 0 -cell $A$ in $\mathbb{A}$.

The 2-cell part sends a modification in the first diagram to the modification with components in the second diagram, for any 0 -cell $A$ in $\mathbb{A}$ :
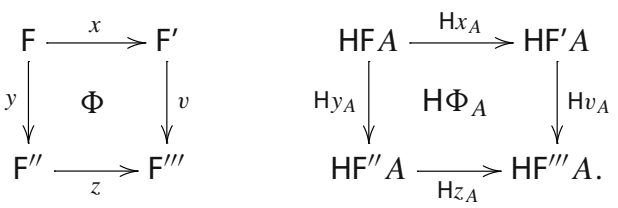

Direct computation verifies the commutativity of

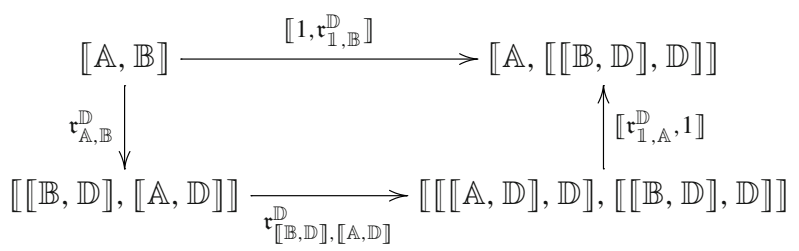

and the equality of

$$
\llbracket \mathbb{A}, \mathbb{D} \rrbracket \stackrel{\mathfrak{r}_{\mathbb{1},}^{\mathbb{D}} \llbracket \mathbb{A}, \mathbb{D} \rrbracket}{\longrightarrow} \llbracket \llbracket \llbracket \mathbb{A}, \mathbb{D} \rrbracket, \mathbb{D} \rrbracket, \mathbb{D} \rrbracket \stackrel{\left[\mathfrak{r}_{\mathbb{1}, \mathbb{A}}, 1 \rrbracket\right.}{\longrightarrow} \llbracket \mathbb{A}, \mathbb{D} \rrbracket
$$

to the identity double functor, for any double categories $\mathbb{A}, \mathbb{B}$ and $\mathbb{D}$. It follows from these properties that the double functors

$$
\mathfrak{f}_{\mathbb{A}, \mathbb{B}}^{\mathbb{D}}=\left(\llbracket \mathbb{A}, \llbracket \mathbb{B}, \mathbb{D} \rrbracket \rrbracket \stackrel{\mathfrak{r}_{\mathbb{A}, \llbracket \mathbb{B}, \mathbb{D} \rrbracket}^{\longrightarrow}}{\longrightarrow} \llbracket \llbracket \llbracket \mathbb{B}, \mathbb{D} \rrbracket, \mathbb{D} \rrbracket, \llbracket \mathbb{A}, \mathbb{D} \rrbracket \rrbracket \stackrel{\llbracket \mathfrak{r}_{\mathbb{1}, \mathbb{B}}^{\mathbb{D}}, 1 \rrbracket}{\longrightarrow} \llbracket \mathbb{B}, \llbracket \mathbb{A}, \mathbb{D} \rrbracket \rrbracket\right)
$$


constitute a natural transformation

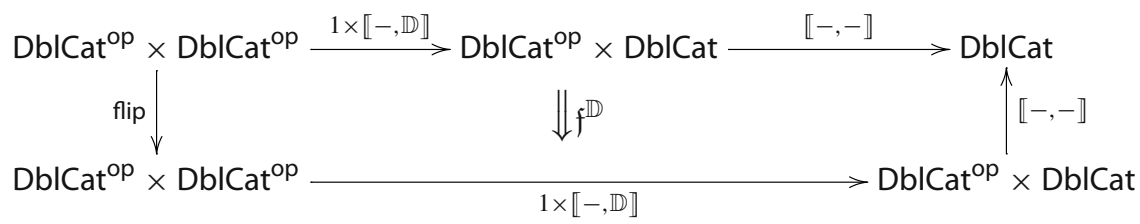

for any double category $\mathbb{D}$, which obeys the idempotent-like property that

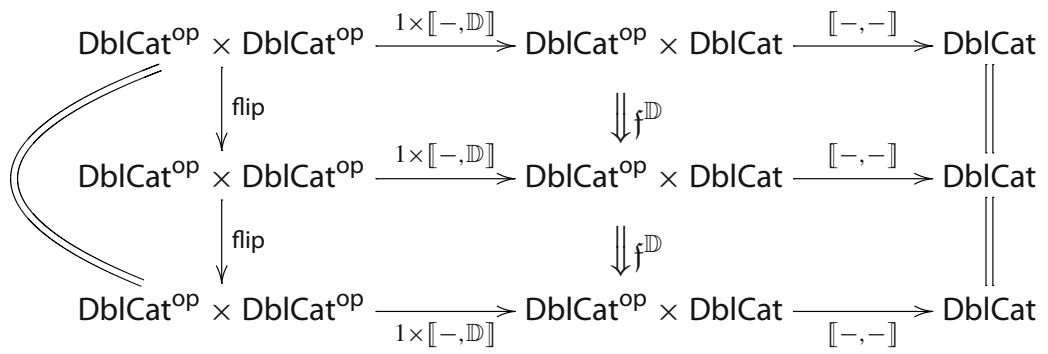

is the identity natural transformation. This $\mathfrak{f}$ is natural in its lower indices by the naturality of $\mathfrak{r}$. It is natural in the upper index as well (here the upper index no longer refers to extranaturality). In order to see that, both naturality and extranaturality of $\mathfrak{r}$ are needed.

The extranatural transformation $\mathfrak{r}$ in this section and $\mathfrak{l}$ in Sect. 2.4 together render commutative the following diagram, for any double categories $\mathbb{A}, \mathbb{B}$ and $\mathbb{D}$.

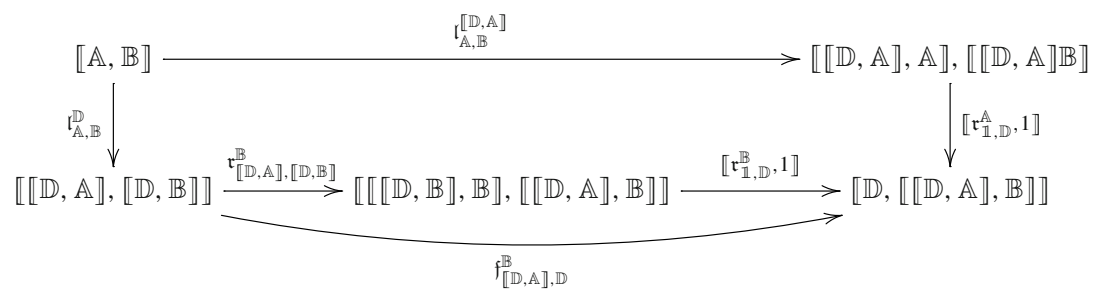

\subsection{Representability of the Functor DbICat $(\mathbb{G}, \llbracket \mathbb{G},-\rrbracket):$ DblCat $\rightarrow$ Set}

In this section we investigate the functor in the title, for the double category $\mathbb{G}$ of Sect. 2.1.

By the description of $\mathbb{G}$ in Sect. 2.1, for any double category $\mathbb{A}$ the double functors $\mathbb{G} \rightarrow \llbracket \mathbb{G}, \mathbb{A} \rrbracket$ correspond bijectively to the 2-cells of $\llbracket \mathbb{G}, \mathbb{A} \rrbracket$. The 0 -cells at the corners of such a 2-cell are double functors denoted as $(A,-): \mathbb{G} \rightarrow \mathbb{A}$, for all 0 -cells $A \in\{X, Y, Z, V\}$ of $\mathbb{G}$. The top and bottom horizontal 1-cells are horizontal pseudo transformations labelled by the horizontal 1 -cells $h \in\{t, b\}$ in $\mathbb{G}$. We denote their components by

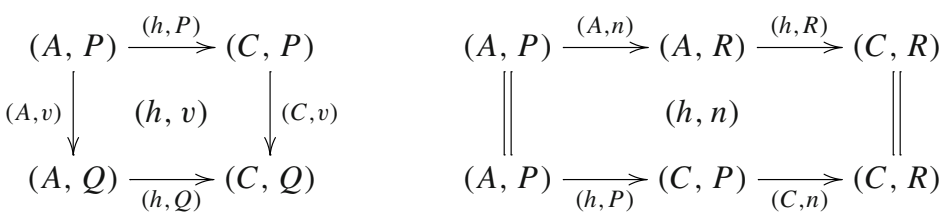


for any horizontal 1 -cell $n$ and vertical 1 -cell $v$ in $\mathbb{G}$. They satisfy the naturality condition

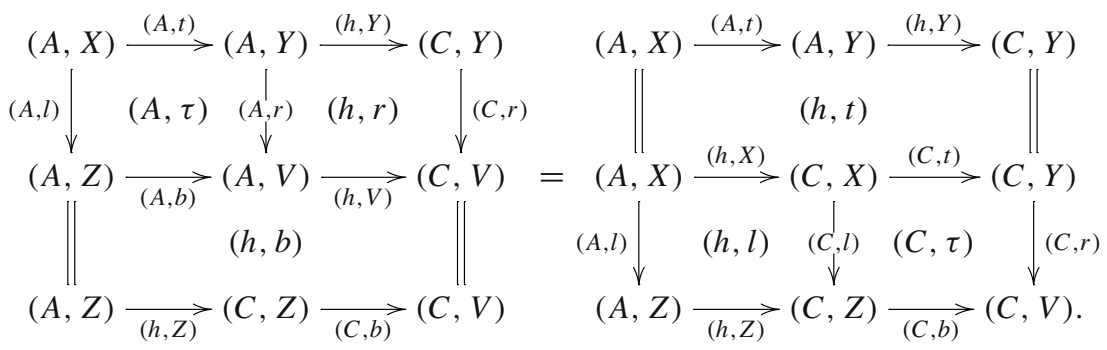

Symmetrically, the left and right vertical 1-cells are vertical pseudo transformations labelled by the vertical 1-cells $w \in\{l, r\}$ of $\mathbb{G}$; with components denoted by
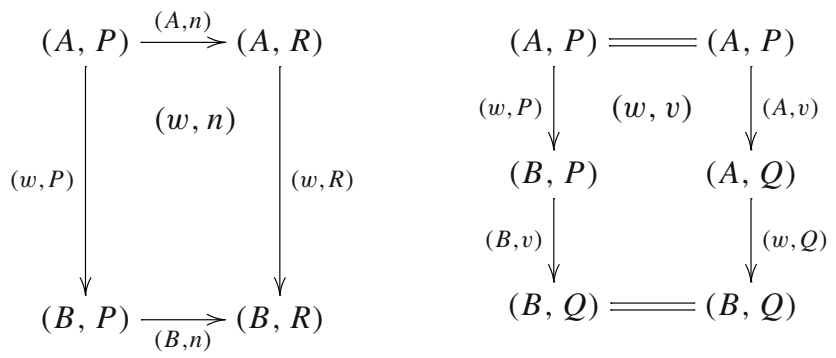

for any horizontal 1 -cell $n$ and vertical 1 -cell $v$ in $\mathbb{G}$. They satisfy the naturality condition

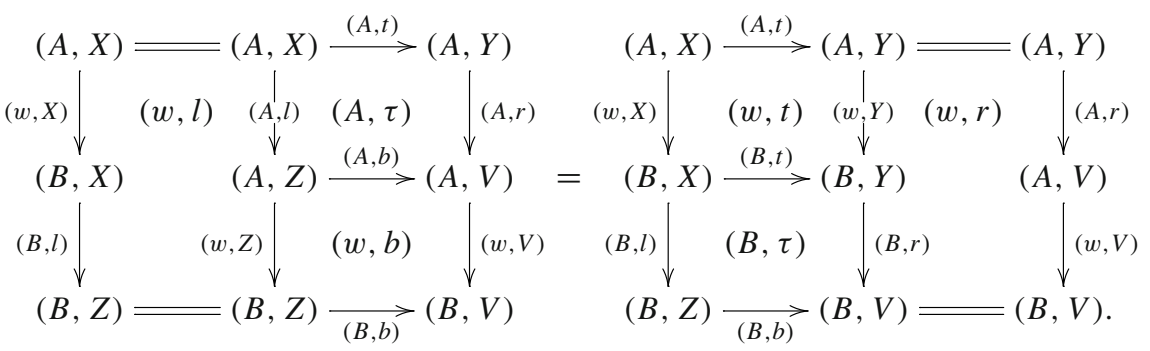

Finally, the 2-cell itself is a modification with components denoted by

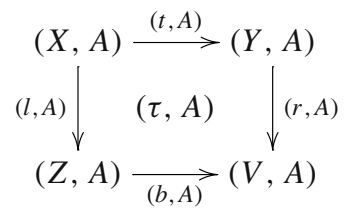


for all 0-cells $A \in\{X, Y, Z, V\}$ of $\mathbb{G}$. They satisfy the horizontal compatibility conditions

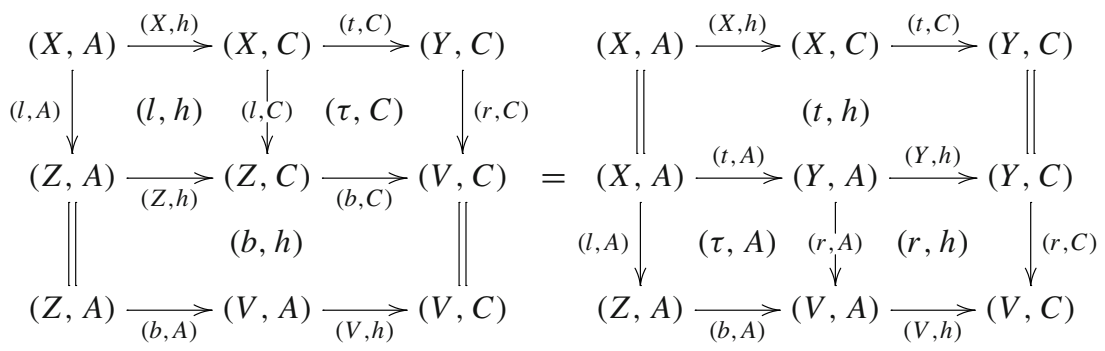

for all horizontal 1-cells $h \in\{t, b\}$ in $\mathbb{G}$; and the vertical compatibility conditions

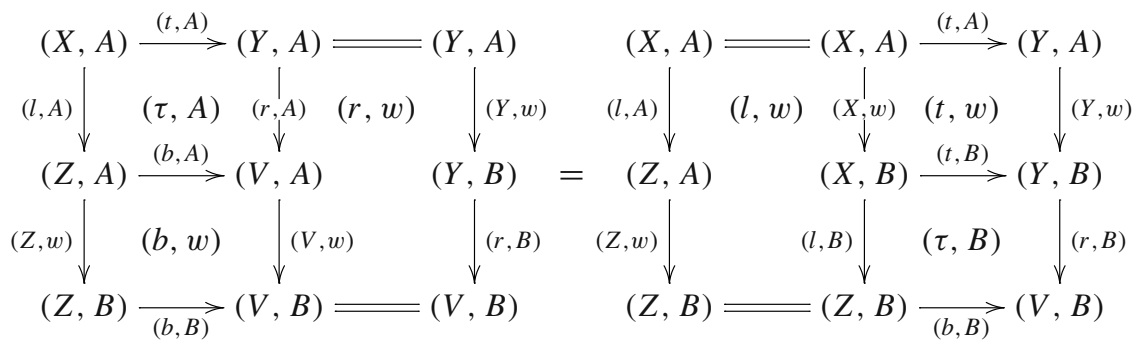

for all vertical 1-cells $w \in\{l, r\}$ in $\mathbb{G}$.

From all that we can read off that the functor DblCat $(\mathbb{G}, \llbracket \mathbb{G},-\rrbracket):$ DblCat $\rightarrow$ Set is represented by the following double category.

- The $\underline{0 \text {-cells }}$ are pairs $(A, B)$ of 0 -cells in $\mathbb{G}$.

- There are two kinds of non-identity horizontal 1-cells $(A, h)$ and $(h, A)$; both are ordered pairs of a 0 -cell $A$, and a horizontal 1-cell $h \in\{t, b\}$ in $\mathbb{G}$.

- Symmetrically, there are two kinds of non-identity vertical 1-cells $(A, v)$ and $(v, A)$; both are ordered pairs of a 0 -cell $A$, and a vertical 1 -cell $v \in\{l, r\}$ in $\mathbb{G}$.

- There are non-identity 2-cells of ordered pairs

- $(A, \tau)$ and $(\tau, A)$, for all 0 -cells $A$ in $\mathbb{G}$

- $(h, v)$ and $(v, h)$ for horizontal 1-cells $h \in\{t, b\}$ and vertical 1-cells $v \in\{l, r\}$ in $\mathbb{G}$

- vertically invertible 2-cells $(h, n)$ for horizontal 1-cells $h, n \in\{t, b\}$ in $\mathbb{G}$

- horizontally invertible 2-cells $(v, w)$ for vertical 1-cells $v, w \in\{l, r\}$ in $\mathbb{G}$.

All further cells are generated by their compositions modulo the associativity and unitality conditions, the middle four interchange law, and the identities (2.5), (2.6), (2.7) and (2.8).

\subsection{Representability of the Functors DbICat $(\mathbb{A}, \llbracket \mathbb{B},-\rrbracket):$ DblCat $\rightarrow$ Set}

In this section we investigate the functors in the title, for any double categories $\mathbb{A}$ and $\mathbb{B}$. Our main tool is the representability result of Sect. 2.6 and the following fact.

Consider a functor $\mathrm{U}: \mathrm{C} \rightarrow \mathrm{C}^{\prime}$ between locally presentable categories. Bourke and Gurski's [6, Lemma 3.9] says that the functor $C^{\prime}(X, \mathrm{U}(-)): C \rightarrow$ Set is representable for all objects $X$-that is, $\mathrm{U}$ possesses a left adjoint — if and only if $\mathrm{C}^{\prime}\left(G_{i}, \mathrm{U}(-)\right): \mathrm{C} \rightarrow$ Set is representable for all $G_{i}$ in a strong generator of $C^{\prime}$. 
The category DblCat is locally presentable by [11, Theorem 4.1]. The double category $\mathbb{G}$ in Sect. 2.6 is a strong generator of DblCat; see Sect. 2.1. So from the representability result of Sect. 2.6 we conclude by Bourke and Gurski's lemma that the functor DblCat $(\mathbb{B}, \llbracket \mathbb{G},-\rrbracket)$ : DblCat $\rightarrow$ Set is representable for any double category $\mathbb{B}$.

The 0 -cell part of the iso double functor (2.3) yields a bijection $\operatorname{DblCat}(\mathbb{B}, \llbracket \mathbb{G}, \mathbb{A} \rrbracket) \cong$ $\operatorname{DblCat}(\mathbb{G}, \llbracket \mathbb{B}, \mathbb{A} \rrbracket)$ for any double categories $\mathbb{A}$ and $\mathbb{B}$; which is natural in $\mathbb{A}$ by the extranaturality and naturality of $\mathfrak{r}$. Therefore also the functor DblCat $(\mathbb{G}, \llbracket \mathbb{B},-\rrbracket):$ DblCat $\rightarrow$ Set is representable for any double category $\mathbb{B}$. Applying again Bourke and Gurski's lemma, we obtain the representability of $\operatorname{DblCat}(\mathbb{A}, \llbracket \mathbb{B},-\rrbracket)$ for any double categories $\mathbb{A}$ and $\mathbb{B}$.

In other words, the functor $\llbracket \mathbb{B},-\rrbracket:$ DblCat $\rightarrow$ DblCat possesses a left adjoint for any double category $\mathbb{B}$ which we denote by $-\otimes \mathbb{B}$. By the functoriality in $\mathbb{B}$, it gives raise to a double functor $\otimes:$ DblCat $\times$ DblCat $\rightarrow$ DblCat. For any double categories $\mathbb{A}$ and $\mathbb{B}$, an explicit description of $\mathbb{A} \otimes \mathbb{B}$ can be given analogously to Sect. 2.6. However, our verification of the coherence of $\otimes$ in Sect. 3 completely avoids using a presentation in terms of generators and relations (which we find an appealing feature). Therefore we do not work out the details of such a presentation.

\section{Coherence}

This section is devoted to the proof that the double functor $\otimes:$ DblCat $\times$ DblCat $\rightarrow$ DblCat of Sect. 2.7 renders DblCat a symmetric monoidal category (which is then closed with the internal hom functors $\llbracket \mathbb{B},-\rrbracket$, for all double categories $\mathbb{B}$ ).

Taking mates under the adjunctions $\mathbb{B} \otimes-\dashv \llbracket \mathbb{B},-\rrbracket$ of Sect. 2 and using the Yoneda Lemma, we relate

- the associativity natural isomorphism to the extranatural transformation $\mathfrak{l}$ of Sect. 2.4;

- the unit natural isomorphisms to the isomorphisms $\mathbb{A} \cong \llbracket \mathbb{1}, \mathbb{A} \rrbracket$ for all double categories $\mathbb{A}$;

- the symmetry to the extranatural transformation $\mathfrak{f}$ of Sect. 2.5.

Then we translate the pentagon, triangle and hexagon conditions to identities on these data.

Morally this is very similar to [8, Proposition 2.3], where for any symmetric closed category in the sense of [8, Definition 1.1] a symmetric closed promonoidal structure is obtained (which is symmetric closed monoidal whenever all internal hom functors are right adjoints). Technically, however, our proof looks smoother than a verification of (DblCat, $\llbracket-,-\rrbracket, \mathbb{1}, \mathbb{B} \cong \llbracket I, \mathbb{B} \rrbracket, 1_{\mathbb{B}}: \mathbb{1} \rightarrow \llbracket \mathbb{B}, \mathbb{B} \rrbracket, \mathfrak{l}, \mathfrak{f}$ ) being a symmetric closed category in the sense of [8, Definition 1.1].

In what follows, the unit of the adjunction $-\otimes \mathbb{B} \dashv \llbracket \mathbb{B},-\rrbracket$ will be denoted by $\eta_{\mathbb{A}}^{\mathbb{B}}$ : $\mathbb{A} \rightarrow \llbracket \mathbb{B}, \mathbb{A} \otimes \mathbb{B} \rrbracket$, and the counit will be denoted by $\epsilon_{\mathbb{A}}^{\mathbb{B}}: \llbracket \mathbb{B}, \mathbb{A} \rrbracket \otimes \mathbb{B} \rightarrow \mathbb{A}$, for all double categories $\mathbb{A}$ and $\mathbb{B}$.

\subsection{The Associativity Natural Isomorphism}

For any double category $\mathbb{C}$, consider the natural transformation 


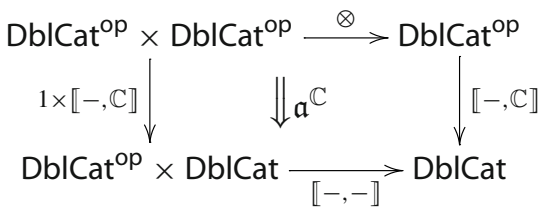

with the components

$$
\mathfrak{a}_{\mathbb{A}, \mathbb{B}}^{\mathbb{C}}:=\left(\llbracket \mathbb{A} \otimes \mathbb{B}, \mathbb{C} \rrbracket \stackrel{\mathfrak{I}_{\mathbb{B} \otimes \mathbb{B}, \mathbb{C}}^{\longrightarrow}}{\longrightarrow} \llbracket \llbracket \mathbb{B}, \mathbb{A} \otimes \mathbb{B} \rrbracket, \llbracket \mathbb{B}, \mathbb{C} \rrbracket \rrbracket \stackrel{\llbracket \eta_{\mathbb{A}}^{\mathbb{B}}, 1 \rrbracket}{\longrightarrow} \llbracket \mathbb{A}, \llbracket \mathbb{B}, \mathbb{C} \rrbracket \rrbracket\right)
$$

at any double categories $\mathbb{A}$ and $\mathbb{B}$. It is natural in $\mathbb{A}$ and $\mathbb{C}$ by the naturality of $\mathfrak{l}$ and $\eta$ (the upper index $\mathbb{C}$ of $\mathfrak{a}$ no longer refers to extranaturality). It is natural in $\mathbb{B}$ as well which follows by the extranaturality of $\mathfrak{l}$ and $\eta$ together with the naturality of $\mathfrak{l}$.

The 0 -cell parts of the iso double functors in (2.3) yield bijections in the columns of the commutative diagram

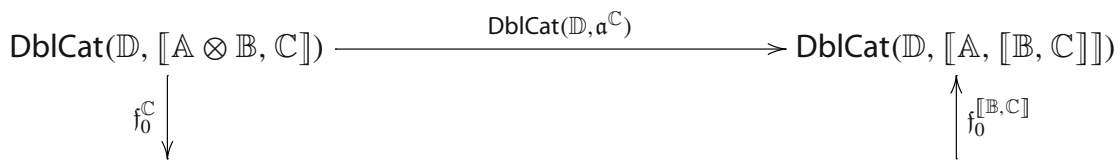

$\operatorname{DblCat}(\mathbb{A} \otimes \mathbb{B}, \llbracket \mathbb{D}, \mathbb{C} \rrbracket) \longrightarrow \operatorname{DblCat}(\mathbb{A}, \llbracket \mathbb{B}, \llbracket \mathbb{D}, \mathbb{C} \rrbracket \rrbracket) \rightarrow \operatorname{DblCat}(\mathbb{A}, \llbracket \mathbb{D}, \llbracket \mathbb{B}, \mathbb{C} \rrbracket \rrbracket)$.

$$
\cong \quad \operatorname{DblCat}\left(\mathbb{A}, f^{\mathbb{C}}\right)
$$

Since all of the occurring maps but the top row are known to be bijections, we conclude that so is the top row. Whence by Yoneda's lemma $\mathfrak{a}^{\mathbb{C}}$ is a natural isomorphism. Using the adjunction isomorphisms in the first and last steps, we obtain a natural isomorphism

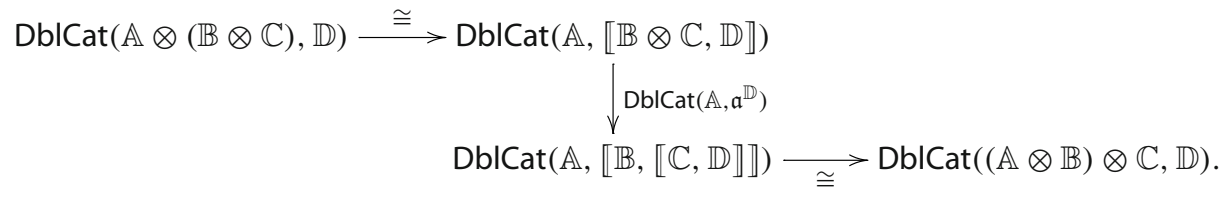

By Yoneda's lemma again, it determines a natural isomorphism $\alpha_{\mathbb{A}, \mathbb{B}, \mathbb{C}}:(\mathbb{A} \otimes \mathbb{B}) \otimes \mathbb{C} \rightarrow$ $\mathbb{A} \otimes(\mathbb{B} \otimes \mathbb{C})$ which is our candidate associativity natural isomorphism.

\subsection{The Pentagon Condition}

By Yoneda's lemma, Mac Lane's pentagon condition on the natural isomorphism $\alpha$ of Sect. 3.1 is equivalent to the commutativity of the exterior of the diagram of Fig. 1; hence also to the commutativity of the diagram of Fig. 2 (the Figures can be found in "Appendix A").

The left column in Fig. 2 is equal to $\llbracket \alpha_{\mathbb{A}, \mathbb{B}, \mathbb{C}}, 1 \rrbracket$. The triangles marked by (*) commute by the naturality of $\mathfrak{l}$ and a triangle condition on the adjunction $-\otimes \mathbb{C} \dashv \llbracket \mathbb{C},-\rrbracket$, yielding the commutative diagram 


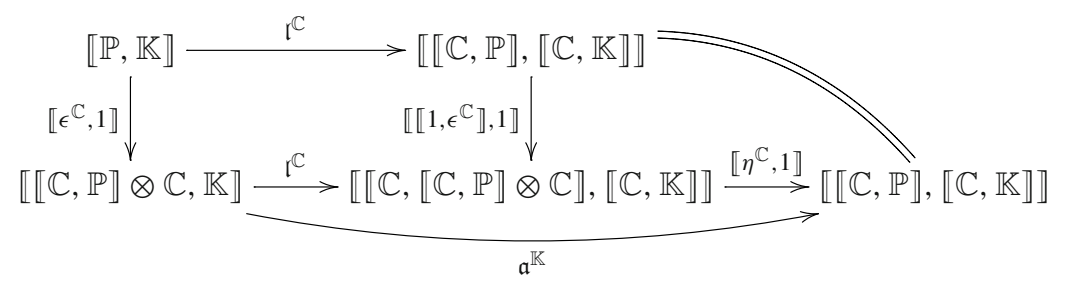

for any double categories $\mathbb{C}, \mathbb{P}$ and $\mathbb{K}$. The region marked by (**) commutes by (2.1) and extranaturality of $\mathfrak{l}$, yielding the commutative diagram

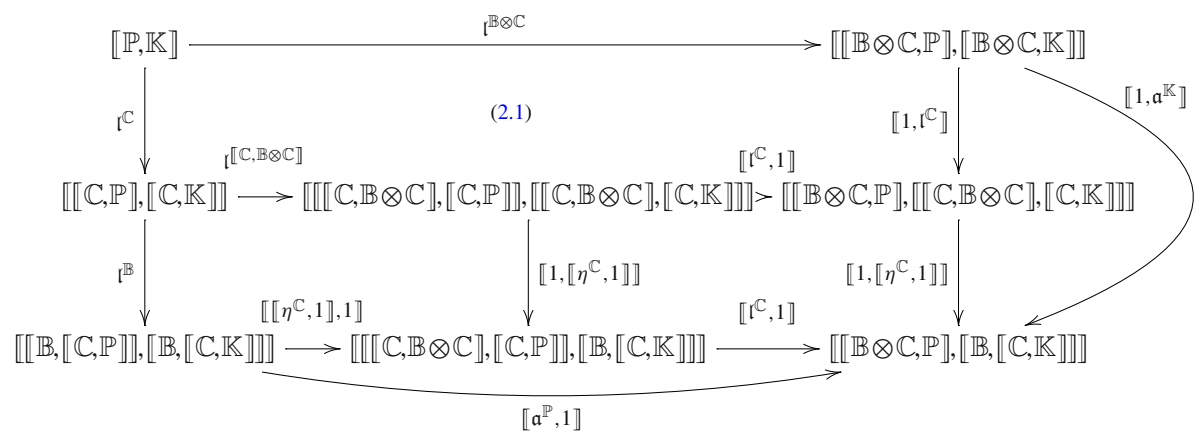

for any double categories $\mathbb{B}, \mathbb{C}, \mathbb{P}$ and $\mathbb{K}$.

\subsection{The Unitality Natural Isomorphisms}

For any double categories $\mathbb{A}$ and $\mathbb{K}$ there are natural isomorphisms

$$
\begin{aligned}
& \operatorname{DblCat}(\mathbb{A}, \mathbb{K}) \cong \operatorname{DblCat}(\mathbb{A}, \llbracket \mathbb{1}, \mathbb{K} \rrbracket) \cong \operatorname{DblCat}(\mathbb{A} \otimes \mathbb{1}, \mathbb{K}) \\
& \operatorname{DblCat}(\mathbb{A}, \mathbb{K}) \cong \operatorname{DblCat}(\mathbb{A}, \llbracket \mathbb{1}, \mathbb{K} \rrbracket) \stackrel{\mathbb{f}_{0}^{\mathbb{K}}}{\longrightarrow} \operatorname{DblCat}(\mathbb{1}, \llbracket \mathbb{A}, \mathbb{K} \rrbracket) \cong \operatorname{DblCat}(\mathbb{1} \otimes \mathbb{A}, \mathbb{K})
\end{aligned}
$$

where $\mathfrak{f}_{0}^{\mathbb{K}}$ denotes the 0 -cell part of the iso double functor (2.3). By Yoneda's lemma, they induce respective natural isomorphisms $\varrho$ and $\lambda$ with the components

$\varrho_{\mathbb{A}}=\left(\mathbb{A} \otimes \mathbb{1} \stackrel{\cong}{\rightarrow} \llbracket \mathbb{1}, \mathbb{A} \rrbracket \otimes \mathbb{1} \stackrel{\epsilon^{\mathbb{1}}}{\rightarrow} \mathbb{A}\right)$ and $\lambda_{\mathbb{A}}=\left(\mathbb{1} \otimes \mathbb{A} \stackrel{1_{A} \otimes 1}{\longrightarrow} \llbracket \mathbb{A}, \mathbb{A} \rrbracket \otimes \mathbb{A} \stackrel{\epsilon^{\mathbb{A}} \rightarrow}{\rightarrow} \mathbb{A}\right)$

at any double category $\mathbb{A}$ (where $1_{A}: \mathbb{1} \rightarrow \llbracket \mathbb{A}, \mathbb{A} \rrbracket$ is the double functor which sends the single object of $\mathbb{1}$ to the identity double functor $1_{A}: \mathbb{A} \rightarrow \mathbb{A}$ ). They are our candidate unitality natural isomorphisms.

\subsection{The Triangle Conditions}

By Yoneda's lemma, Mac Lane's triangle condition on the natural isomorphisms $\alpha$ of Sect. 3.1 and $\lambda, \varrho$ of Sect. 3.3 is equivalent to the commutativity of the exterior of Fig. 3 (see "Appendix A") for any double categories $\mathbb{A}, \mathbb{B}$ and $\mathbb{K}$; hence also to the commutativity of 


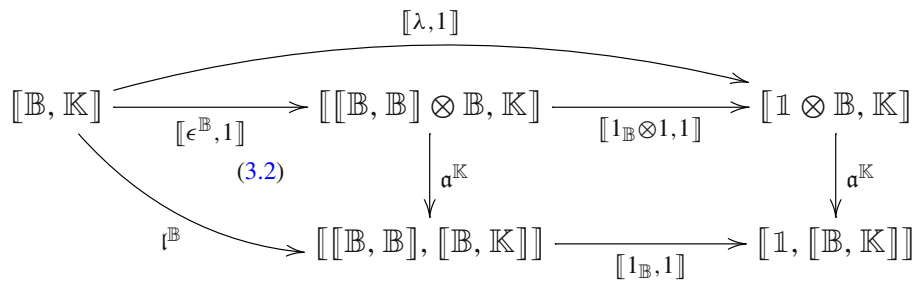

whose left-bottom path is (2.2); that is, the canonical (usually omitted) isomorphism.

\subsection{The Symmetry}

The natural isomorphism

$\operatorname{DblCat}(\mathbb{B} \otimes \mathbb{A}, \mathbb{K}) \stackrel{\cong}{\longrightarrow} \operatorname{DblCat}(\mathbb{B}, \llbracket \mathbb{A}, \mathbb{K} \rrbracket) \stackrel{f_{0}^{\mathbb{K}}}{\longrightarrow} \operatorname{DblCat}(\mathbb{A}, \llbracket \mathbb{B}, \mathbb{K} \rrbracket) \stackrel{\cong}{\longrightarrow} \operatorname{DblCat}(\mathbb{A} \otimes \mathbb{B}, \mathbb{K})$ constructed from the 0 -cell part of $\mathfrak{f}$ in (2.3) induces a natural isomorphism $\varphi: \otimes \rightarrow \otimes$.flip with the components

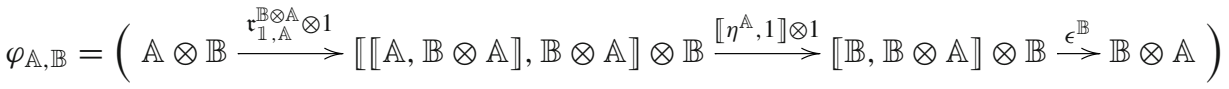
at any double categories $\mathbb{A}$ and $\mathbb{B}$. It is our candidate symmetry.

\subsection{The Hexagon Condition}

By Yoneda's lemma, the hexagon condition on the natural isomorphisms $\alpha$ of Sect. 3.1 and $\varphi$ of Sect. 3.5 is equivalent to the commutativity of the exterior of Fig. 4 for any double categories $\mathbb{A}, \mathbb{B}, \mathbb{C}$ and $\mathbb{K}$. Hence it follows by the commutativity of the diagram of Fig. 5 whose left-bottom path is equal to $\mathfrak{a}^{\mathbb{K}}$. (The Figures can be found in "Appendix A".)

In order to see that the left column of Fig. 5 is equal to $\mathfrak{I}^{\mathbb{A}}$, apply twice (2.4) to obtain the commutative diagram

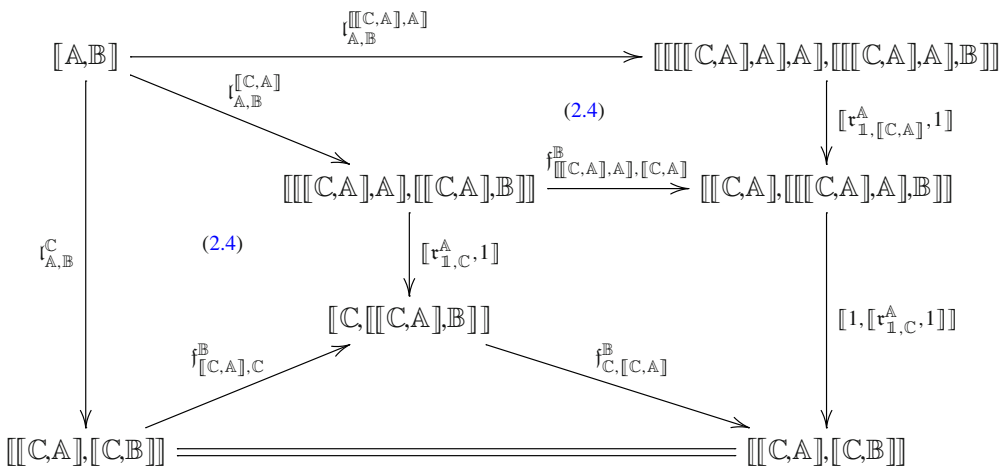

for any double categories $\mathbb{A}, \mathbb{B}$ and $\mathbb{C}$. The region of Fig. 5 marked by $(*)$ commutes by the extranaturality of $\mathfrak{l}$. 


\section{Examples}

Obviously, what we get for the closed Gray monoidal product $\otimes$ of double categories essentially depends on our choice of the internal hom double categories $\llbracket \mathbb{A}, \mathbb{B} \rrbracket$. Although our notions of (horizontal and vertical) pseudo transformations and of corresponding modification in Sect. 2.2 may look quite natural, admittedly no higher principle fixes their choice. Therefore there is no a'priori good resulting Gray monoidal product of double categories. In this final section we support our construction by relating it to existing structures. Namely, we verify the monoidality of some well-known functors between our monoidal category $($ DblCat, $\otimes)$ and some other monoidal categories which occur in the literature quite frequently.

\subsection{Monoidal Functors Between Closed Monoidal Categories}

In any closed monoidal category we may take the mate

$$
\begin{gathered}
\mathfrak{a}_{A, B}^{C}:=\left([A \otimes B, C] \stackrel{\eta^{A}}{\longrightarrow}[A,[A \otimes B, C] \otimes A] \stackrel{\left[1, \eta^{B}\right]}{\longrightarrow}[A,[B,([A \otimes B, C] \otimes A) \otimes B] \stackrel{[1,[1, \alpha]]}{\longrightarrow}\right. \\
{\left[A,\left[B,[A \otimes B, C] \otimes(A \otimes B) \rrbracket \stackrel{\left[1,\left[1, \epsilon^{A \otimes B}\right]\right]}{\longrightarrow}[A,[B, C]]\right)\right.}
\end{gathered}
$$

of the associativity isomorphism $\alpha$ under the adjunctions $-\otimes X \dashv[X,-]$ for $X$ being the objects $A, B$ and $A \otimes B$; and its mate

$$
\mathfrak{l}_{A, B}^{C}:=\left(\left([A, B] \stackrel{\left[\epsilon^{C}, 1\right]}{\longrightarrow}[[C, A] \otimes C, B] \stackrel{\mathfrak{a}^{B}}{\longrightarrow}[[C, A],[C, B]]\right) .\right.
$$

Consider now a functor $\mathrm{H}$ between closed monoidal categories. Some natural transformation $\mathrm{H}_{2}: \mathrm{H}(-) \otimes \mathrm{H}(-) \rightarrow \mathrm{H}(-\otimes-$ ) (for both monoidal products denoted by $\otimes$ ) and a morphism $\mathrm{H}_{0}: I \rightarrow \mathrm{H} I$ (for both monoidal units denoted by $I$ ) render $\mathrm{H}$ monoidal if and only if the mate

$$
\chi_{A, B}:=\left(\mathrm{H}[A, B] \stackrel{\eta^{\mathrm{H} A}}{\rightarrow}[\mathrm{H} A, \mathrm{H}[A, B] \otimes \mathrm{H} A] \stackrel{\left[1, \mathrm{H}_{2}\right]}{\longrightarrow}[\mathrm{H} A, \mathrm{H}([A, B] \otimes A)] \stackrel{\left[1, \mathrm{H} \epsilon^{A}\right]}{\longrightarrow}[\mathrm{H} A, \mathrm{H} B]\right)
$$

of $\mathrm{H}_{2}$ under the adjunctions $-\otimes A \dashv[A,-]$ and $-\otimes \mathrm{H} A \dashv[\mathrm{H} A,-]$ makes the following diagrams commute.

The left and right unitality conditions translate to the commutativity of the respective diagrams in
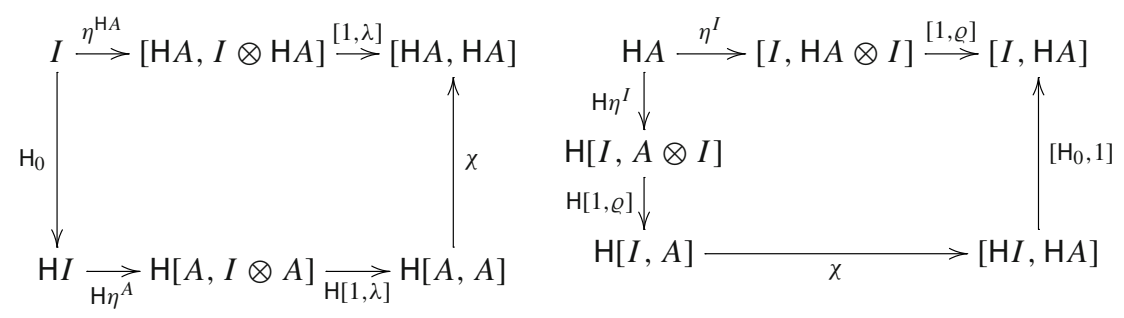
for all objects $A$ of the domain category. The associativity condition translates to the commutativity of

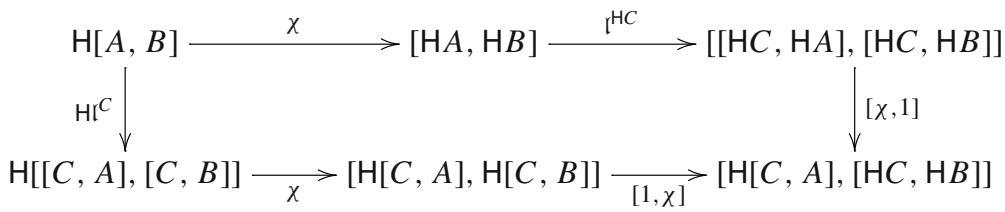

for all objects $A, B$ and $C$ of the domain category.

\subsection{The Closed Monoidal Category (DbICat, $\otimes)$}

For the closed monoidal category (DblCat, $\otimes$ ) of Sects. 2 and 3,

$$
\mathbb{1} \stackrel{\eta^{\mathbb{A}}}{\longrightarrow} \llbracket \mathbb{A}, \mathbb{1} \otimes \mathbb{A} \rrbracket \stackrel{[1, \lambda \rrbracket}{\longrightarrow} \llbracket \mathbb{A}, \mathbb{A} \rrbracket
$$

is equal to $1_{\mathbb{A}}$; that is, the double functor sending the single object of $\mathbb{1}$ to the identity double functor $1_{\mathbb{A}}: \mathbb{A} \rightarrow \mathbb{A}$ for any double category $\mathbb{A}$. The double functor

$$
\mathbb{A} \stackrel{\eta^{\mathbb{1}}}{\longrightarrow} \llbracket \mathbb{1}, \mathbb{A} \otimes \mathbb{1} \rrbracket \stackrel{\llbracket 1, \varrho \rrbracket}{\longrightarrow} \llbracket \mathbb{1}, \mathbb{A} \rrbracket
$$

is the canonical isomorphism for any double category $\mathbb{A}$. The double functor of (4.1) is equal to that in Sect. 2.4.

\subsection{Monoids in (DblCat, $\otimes)$}

Monoidal 2-categories can be defined at different levels of generality. The most restrictive one in the literature is a monoid in the category of 2-categories and 2-functors with respect to the Cartesian monoidal structure. This is known as a strict monoidal 2-category. The most general one is a single object tricategory [14]; known as a monoidal bicategory. In between them are the so-called Gray monoids; these are again monoids in the category of 2-categories and 2 -functors, but in this case with respect to the Gray monoidal structure [19]. Their importance stems from the coherence result of [14], proving that any monoidal bicategory is equivalent to a Gray monoid (as a tricategory).

Analogously, a strict monoidal double category [7] is a monoid in the category of double categories and double functors with respect to the Cartesian monoidal structure. In [18,24] it was generalized to a pseudo monoid in the 2-category of (pseudo) double categories and pseudo double functors and, say, vertical transformations. However, no double category analogues of Gray monoids and of monoidal bicategories seem to be available in the literature. While the considerations in this paper do not promise any insight how to define most general monoidal (pseudo) double categories, monoids in (DblCat, $\otimes$ ) are natural candidates for the double category analogue of Gray monoid. In this section we give their explicit characterization, similar to the characterization of Gray monoids in [3, Lemma 4].

A monoid in (DblCat, $\otimes$ ) is equivalently a monoidal functor from the terminal double category $\mathbb{1}$ (with the trivial monoidal structure) to (DbICat, $\otimes$ ). It can be described in terms of the data in Sect. 4.1. Namely, a monoid structure on a double category $\mathbb{A}$ translates to double functors $I: \mathbb{1} \rightarrow \mathbb{A}$ and $M: \mathbb{A} \rightarrow \llbracket \mathbb{A}, \mathbb{A} \rrbracket$ which render commutative the diagrams of (4.2) and (4.3). Spelling out the details, this amounts to the following data. 
- A distinguished 0-cell $I$.

- For any 0-cells $X$ and $Y$, a 0 -cell $X * Y$.

- For any 0-cell $Y$ and any horizontal 1-cell on the left below, horizontal 1-cells on the right:

$$
X \stackrel{h}{\longrightarrow} X^{\prime} \quad X * Y \stackrel{h * Y}{\longrightarrow} X^{\prime} * Y \text { and } Y * X \stackrel{Y * h}{\longrightarrow} Y * X^{\prime} .
$$

- For any 0-cell $Y$ and any vertical 1-cell on the left below, vertical 1-cells on the right:

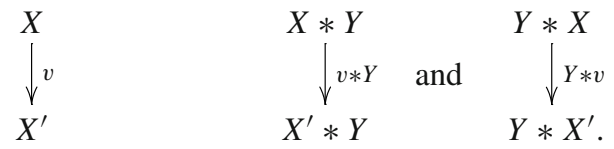

- For any 0-cell $Y$ and any 2-cell on the left below, 2-cells on the right:
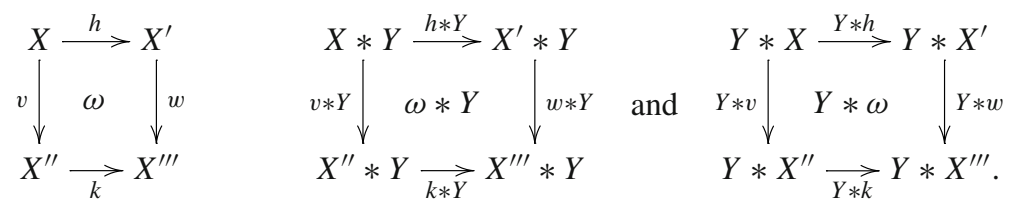

- For any horizontal 1-cell $h$ and any vertical 1-cell $q, 2$-cells

$$
\begin{aligned}
& X * Y \stackrel{h * Y}{\longrightarrow} X^{\prime} * Y \quad \text { and } \quad Y * X \stackrel{Y * h}{\longrightarrow} Y * X^{\prime}
\end{aligned}
$$

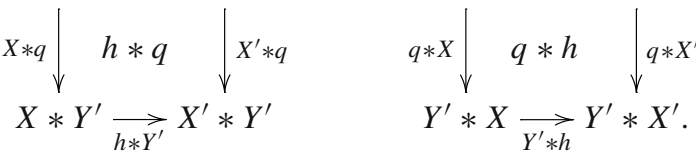

- For any horizontal 1-cells $h$ and $p$, a vertically invertible 2-cell

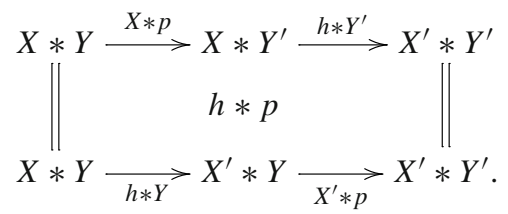

- For any vertical 1-cells $v$ and $q$, a horizontally invertible 2-cell

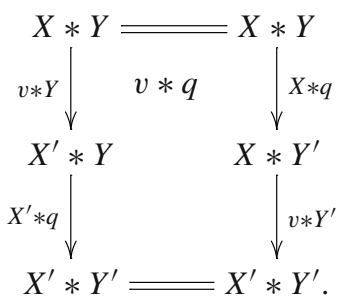

One can memorize this as the rule that a pair of an $n$ dimensional and an $m$ dimensional cell is sent by the operation $*$ to an $n+m \leq 2$ dimensional cell. These data are subject to the following conditions.

(i) For any 0 -cell $X, X *-$ and $-* X$ are double functors $\mathbb{A} \rightarrow \mathbb{A}$.

(ii) $I *-=1_{\mathbb{A}}=-* I$. 
(iii) For any 0-cells $X$ and $Y$, the following equalities of double functors hold.

$$
X *(Y *-)=(X * Y) *-\quad X *(-* Y)=(X *-) * Y \quad-*(X * Y)=(-* X) * Y
$$

(iv) For any 0 -cell $X$, any horizontal 1 -cells $h, p$ and any vertical 1 -cells $v, q$, the following equalities of 2-cells hold.

$$
\begin{aligned}
& h *(X * q)=(h * X) * q(X * h) * q=X *(h * q) \quad h *(q * X)=(h * q) * X \\
& v *(X * p)=(v * X) * p(X * v) * p=X *(v * p) \quad v *(p * X)=(v * p) * X \\
& h *(X * p)=(h * X) * p(X * h) * p=X *(h * p) h *(p * X)=(h * p) * X \\
& v *(X * q)=(v * X) * q(X * v) * q=X *(v * q) \quad v *(q * X)=(v * q) * X
\end{aligned}
$$

(v) For any 0 -cell $X$, we denote by $1_{X}$ the horizontal identity 1 -cell; and by $1^{X}$ the vertical identity 1 -cell on $X$. For any horizontal 1 -cell $h$ and any vertical 1 -cell $v$, the following equalities of 2-cells hold.

$$
\begin{aligned}
& h * 1^{X}=\|\underset{h * X}{\stackrel{h * X}{\longrightarrow}}\|=h * 1_{X} \text { and } v * 1_{X}=v * X \mid \overline{\overline{\underbrace{}_{1}}} v * X=v * 1^{X}
\end{aligned}
$$

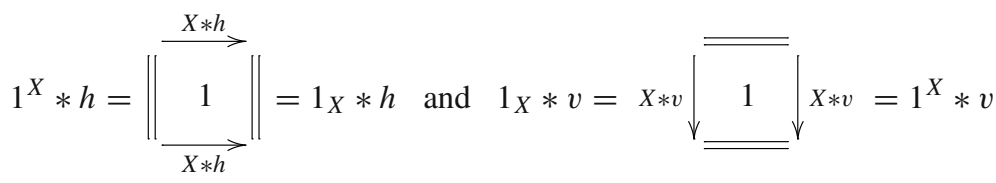

(vi) We denote the vertical composition (of vertical 1-cells and of 2-cells) by an upper dot, and the horizontal composition (of horizontal 1-cells and of 2-cells) by a lower dot. For any composable pairs of horizontal 1-cells $h, h^{\prime}$ and $p, p^{\prime}$ and for any composable pairs of vertical 1-cells $v, v^{\prime}$ and $q, q^{\prime}$, the following hold.

$h *\left(q^{\prime} q\right)=\left(h * q^{\prime}\right)^{\cdot}(h * q)$ and $\left(h^{\prime} . h\right) * q=\left(h^{\prime} * q\right) .(h * q)$

$v *\left(p^{\prime} \cdot p\right)=\left(v * p^{\prime}\right) .(v * p)$ and $\left(v^{\prime} v\right) * p=\left(v^{\prime} * p\right)^{\cdot}(v * p)$

$h *\left(p^{\prime} . p\right)$ and $\left(h^{\prime} . h\right) * p$ are equal to the respective 2-cells
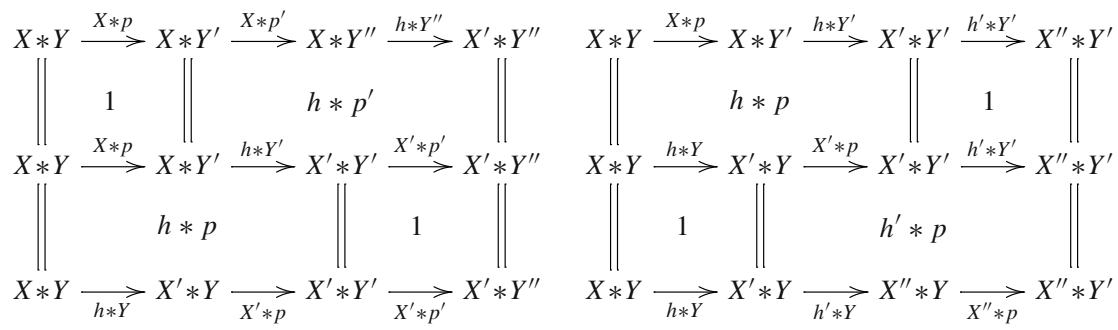
$v *\left(q^{\prime} q\right)$ and $\left(v^{\prime} v\right) * q$ are equal to the respective 2-cells

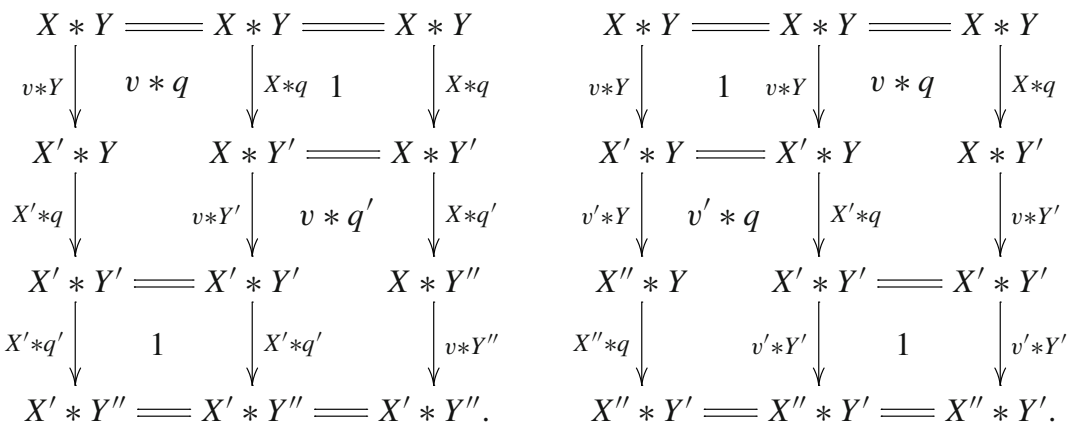

(vii) For every 2-cell $\omega$, horizontal 1 -cell $h$ and vertical 1 -cell $v$, the following naturality conditions hold.

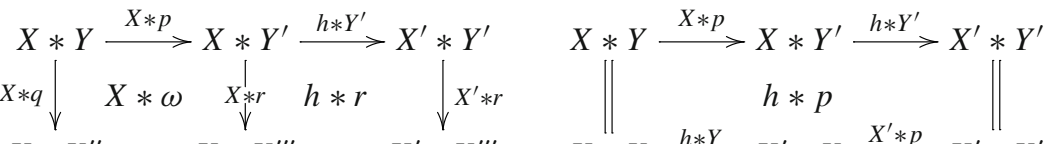

$$
\begin{aligned}
& X * Y^{\prime \prime} \underset{X * s}{\longrightarrow} X * Y^{\prime \prime \prime} \underset{h * Y^{\prime \prime \prime}}{\stackrel{\longrightarrow}{\longrightarrow}} X^{\prime} * Y^{\prime \prime \prime}=X * Y \stackrel{h * Y}{\longrightarrow} X^{\prime} * Y \stackrel{X^{\prime} * p}{\longrightarrow} X^{\prime} * Y^{\prime} \\
& X * Y^{\prime \prime} \underset{h * Y^{\prime \prime}}{\longrightarrow} X^{\prime} * Y^{\prime \prime} \underset{X^{\prime} * s}{\longrightarrow} X^{\prime} * Y^{\prime \prime \prime} \quad X * Y^{\prime \prime} \underset{h * Y^{\prime \prime}}{\longrightarrow} X^{\prime} * Y^{\prime \prime} \underset{X^{\prime} * s}{\longrightarrow} X^{\prime} * Y^{\prime \prime \prime}
\end{aligned}
$$

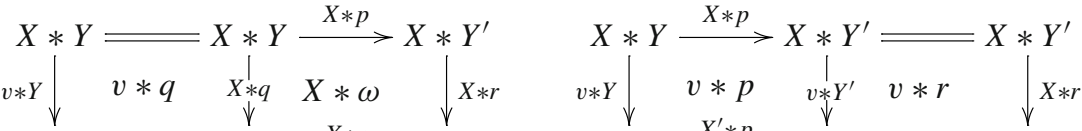

$$
\begin{aligned}
& X^{\prime} * Y \quad X * Y^{\prime \prime} \stackrel{X * s}{\longrightarrow} X * Y^{\prime \prime \prime}=X^{\prime} * Y \stackrel{X^{\prime} * p}{\longrightarrow} X^{\prime} * Y^{\prime} \quad X * Y^{\prime \prime \prime} \\
& X^{\prime} * q \downarrow \quad v * Y^{\prime \prime} \downarrow \quad v * s \quad \downarrow * Y^{\prime \prime \prime} \quad X^{\prime} * q \downarrow \quad X^{\prime} * \omega \quad \downarrow X^{\prime} * r \quad \downarrow v * Y^{\prime \prime \prime} \\
& X^{\prime} * Y^{\prime \prime}=X^{\prime} * Y^{\prime \prime} \underset{X^{\prime} * S}{\longrightarrow} X^{\prime} * Y^{\prime \prime \prime} \quad X^{\prime} * Y^{\prime \prime} \underset{X^{\prime} * S}{\longrightarrow} X^{\prime} * Y^{\prime \prime \prime}=X^{\prime} * Y^{\prime \prime \prime} \\
& Y * X \stackrel{Y * h}{\longrightarrow} Y * X^{\prime} \stackrel{p * X^{\prime}}{\longrightarrow} Y^{\prime} * X^{\prime} \quad Y * X \stackrel{Y * h}{\longrightarrow} Y * X^{\prime} \stackrel{p * X^{\prime}}{\longrightarrow} Y^{\prime} * X^{\prime} \\
& q * X\left|q * h \quad \underset{\substack{q * X^{\prime} \\
\forall}}{\mid} \omega * X^{\prime} \quad \downarrow^{r * X^{\prime}} \quad \| \quad p * h \quad\right| \\
& Y^{\prime \prime} * X \underset{Y^{\prime \prime} * h}{\longrightarrow} Y^{\prime \prime} * X^{\prime} \underset{s * X^{\prime}}{\longrightarrow} Y^{\prime \prime \prime} * X^{\prime}=Y * X \stackrel{p * X}{\longrightarrow} Y^{\prime} * X \stackrel{Y^{\prime} * h}{\longrightarrow} Y^{\prime} * X^{\prime} \\
& Y^{\prime \prime} * X \underset{s * X}{\underset{Y^{\prime \prime \prime}}{\longrightarrow}} Y^{\prime \prime \prime} * X \underset{Y^{\prime \prime \prime}}{\longrightarrow} Y^{\prime \prime \prime} X^{\prime} \quad Y^{\prime \prime} * X \underset{s * X}{\longrightarrow} Y^{\prime \prime \prime} * X \underset{Y^{\prime \prime \prime} * h}{\longrightarrow} Y^{\prime \prime \prime} * X^{\prime}
\end{aligned}
$$




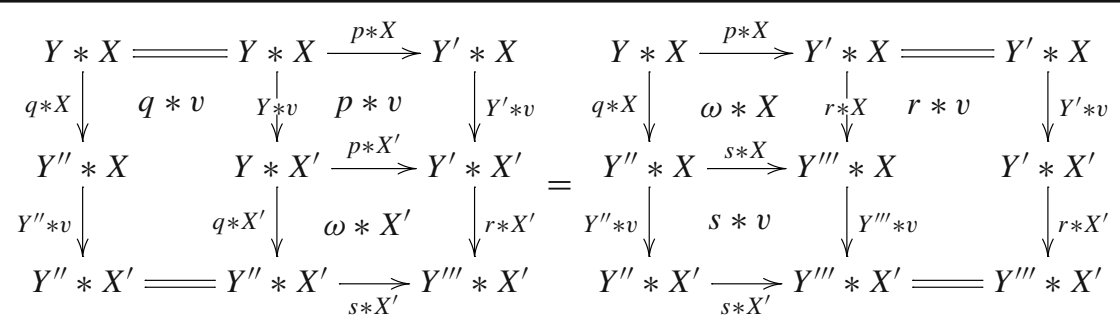

In part (vii) we see the naturality conditions on the horizontal and vertical pseudo transformations obtained as the images of 1-cells under the double functor $M: \mathbb{A} \rightarrow \llbracket \mathbb{A}, \mathbb{A} \rrbracket$; and the compatibility conditions on the modifications obtained as the images of 2-cells under $M$. The conditions in (i-v-vi) come from two sources: from the requirements that $M$, and the image of any 0-cell under it, are double functors; and from the functoriality conditions on the horizontal and vertical pseudo transformations obtained as the images of 1-cells under $M$. Condition (i) expresses the unitality, and (iii-iv) express the associativity of the monoid.

Using the notation of this section, one can easily introduce multiplication maps $\circledast$ : $\mathbb{A} \times \mathbb{A} \rightarrow \mathbb{A}$ on the various cells. They are associative with the unit $I$ :

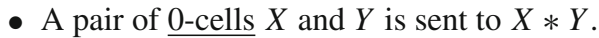

- A pair of horizontal 1-cells $h$ and $k$ is sent to the horizontal 1-cell on the left; and a pair of vertical 1-cells $f$ and $g$ is sent to the vertical 1-cell on the right:

$$
X * Y \stackrel{h * Y}{\longrightarrow} X^{\prime} * Y \stackrel{X^{\prime} * k}{\longrightarrow} X^{\prime} * Y^{\prime} \quad \begin{gathered}
X * Y \\
\downarrow \\
X^{\prime} * Y * Y \\
\downarrow \\
X^{\prime} * X^{\prime} * g
\end{gathered}
$$

- A pair of 2-cells $\omega$ and $\vartheta$ is sent to

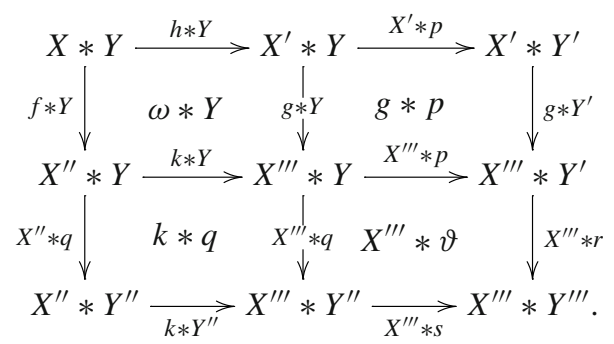

These maps are checked to constitute a double pseudo functor $\circledast: \mathbb{A} \times \mathbb{A} \rightarrow \mathbb{A}$ in the sense of [23, Definition 6.1] with trivial nullary parts and horizontal, respectively, vertical binary parts 

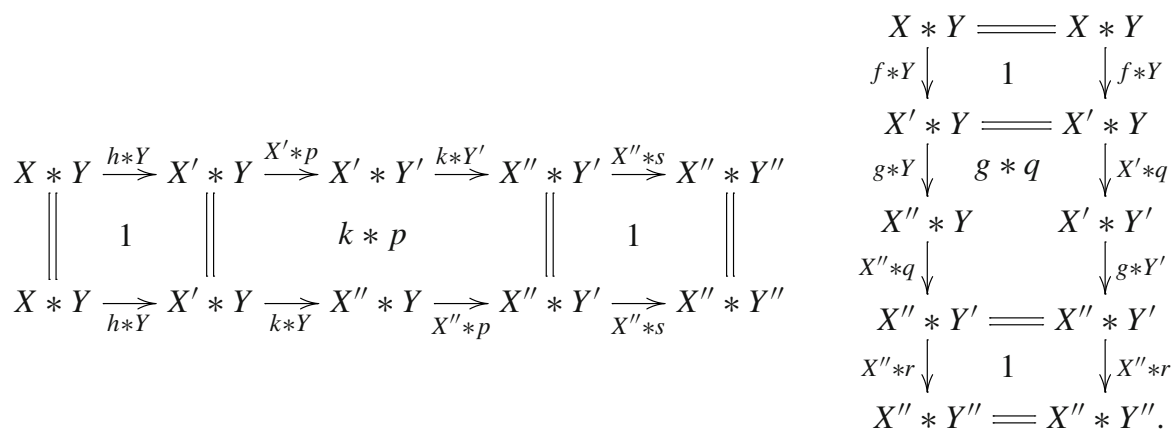

Consequently, monoids in (DblCat, $\otimes$ ) in this section determine monoids-with this multiplication $\circledast$-in the category whose objects are the double categories, whose morphisms are the double pseudo functors of [23, Definition 6.1], and which is monoidal via the Cartesian product.

However, the multiplication $\circledast$ of this monoid need not be a double functor or at least a pseudo double functor in the sense of [16, Section 2.1] and [24, Definition 2.7] (recall that a pseudo double functor in these references is defined to strictly preserve the composition in one direction; and up-to a coherent natural family of invertible 2-cells in the other direction). Consequently, there seems to be no evident way to interpret a monoid in (DblCat, $\otimes$ ) like above as a monoidal double category in the sense of [24, Definition 2.9], [18, Section 3.1], [12, Definition 31] and [16, Section 5.5]; or-what is the same-as a one object locally cubical bicategory in [13]. By this reason, there seems to be no easy way to regard a monoid in $($ DblCat, $\otimes)$ in this section as a suitably degenerate intercategory $[17,18]$.

\subsection{Monoidality of the Functor Mnd : $($ DblCat, $\otimes) \rightarrow($ DblCat, $\otimes)$ Due to Fiore, Gambino and Kock [10]}

To any double category $\mathbb{A}$, the double category $\mathbb{M} n(\mathbb{A})$ of monads in $\mathbb{A}$ was associated in [10]. This construction can be seen as the object map of the functor in the title, which sends a morphism; that is, a double functor $F: \mathbb{A} \rightarrow \mathbb{B}$ to the double functor $\mathbb{M n d}(F): \mathbb{M n d}(\mathbb{A}) \rightarrow$ $\mathbb{M n d}(\mathbb{B})$ of 'componentwise' action.

As the nullary part of the candidate monoidal structure, we take the evident iso double functor $\mathbb{1} \stackrel{\cong}{\cong} \mathbb{n d}(\mathbb{1})$. For any double categories $\mathbb{A}$ and $\mathbb{B}$, for the double functors $\chi_{\mathbb{A}, \mathbb{B}}$ : $\mathbb{M n d} \llbracket \mathbb{A}, \mathbb{B} \rrbracket \rightarrow \llbracket \mathbb{M n d}(\mathbb{A}), \mathbb{M n d}(\mathbb{B}) \rrbracket$ encoding the binary part, we propose the following.

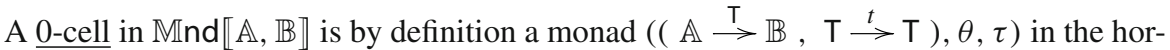
izontal 2-category of $\llbracket \mathbb{A}, \mathbb{B} \rrbracket$. We have to associate to it a 0 -cell in $\llbracket \mathbb{M n d}(\mathbb{A}), \mathbb{M n d}(\mathbb{B}) \rrbracket$; that is, a double functor $\chi_{\mathbb{A}, \mathbb{B}}((\mathrm{T}, t), \theta, \tau): \mathbb{M n d}(\mathbb{A}) \rightarrow \mathbb{M n d}(\mathbb{B})$.

Evaluation at any 0 -cell $X$ of $\mathbb{A}$ gives a 0 -cell $\left(\left(\mathrm{T} X, t_{X}\right), \theta_{X}, \tau_{X}\right)$ in $\mathbb{M n d}(\mathbb{B})$. The image of any 0 -cell $((X, x), \mu, \eta)$ of $\mathbb{M n d}(\mathbb{A})$ under the double functor $\mathbb{M n d}(\mathrm{T})$ is a 0 cell $((\mathrm{T} X, \mathrm{~T} x), \mathrm{T} \mu, \mathrm{T} \eta)$ in $\mathbb{M n d}(\mathbb{B})$. Between these monads in the horizontal 2-category of $\mathbb{B}$, there is a distributive law $t^{x}$. It induces a 0 -cell in $\mathbb{M n d}(\mathbb{B})$, 


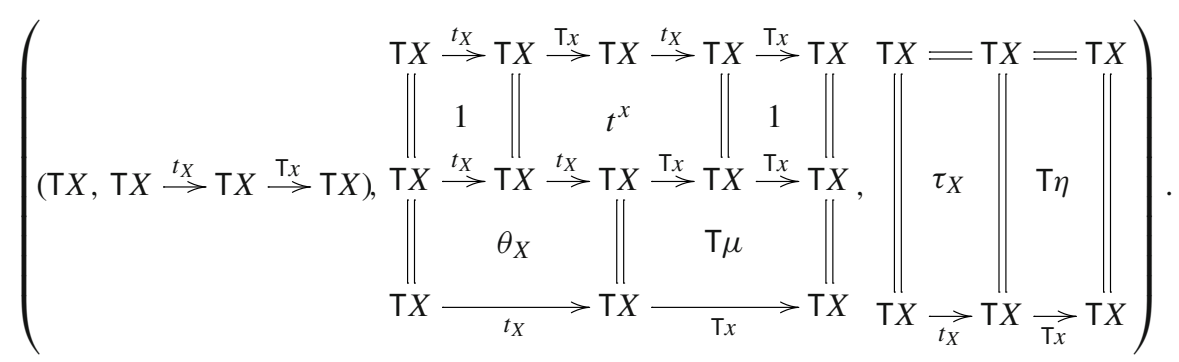

It will be the image of the 0 -cell $((X, x), \mu, \eta)$ of $\mathbb{M n d}(\mathbb{A})$ under the double functor

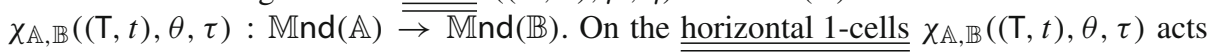
as

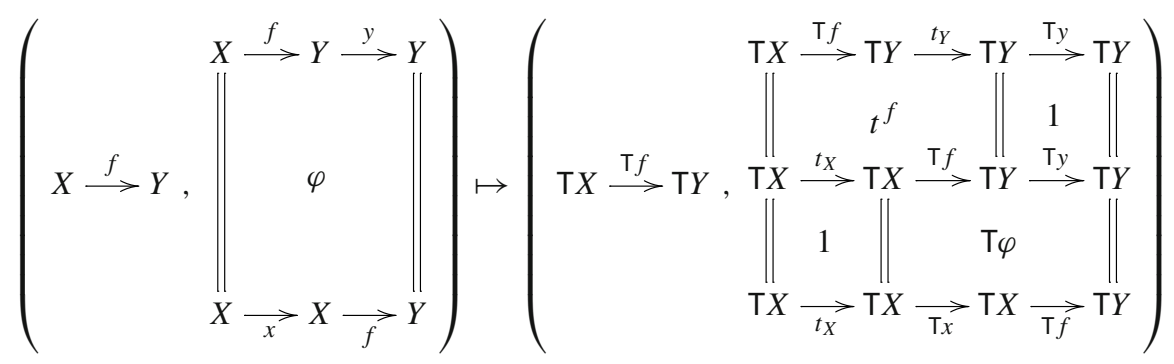

and on the vertical 1-cells it acts as

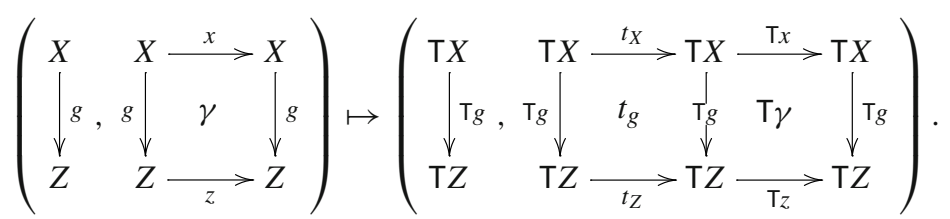

Finally, $\chi_{\mathbb{A}, \mathbb{B}}((\mathrm{T}, t), \theta, \tau)$ sends a 2-cell $\omega$ in $\mathbb{M n d}(\mathbb{A})$ to the 2-cell $\mathrm{T} \omega$ of $\mathbb{M} n d(\mathbb{B})$.

A horizontal 1-cell in $\mathbb{M n d} \llbracket \mathbb{A}, \overline{\mathbb{B} \rrbracket}$ is a monad morphism

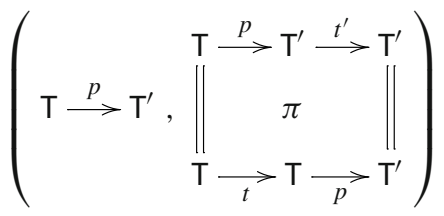

in the horizontal 2-category of $\llbracket \mathbb{A}, \mathbb{B} \rrbracket$ (so that in particular $p$ is a horizontal pseudo transformation and $\pi$ is a modification). The double functor $\chi_{\mathbb{A}, \mathbb{B}}$ should send it to a horizontal 1-cell in $\llbracket \mathbb{M n d}(\mathbb{A}), \mathbb{M n d}(\mathbb{B}) \rrbracket$; that is, the following horizontal pseudo transformation $\chi_{\mathbb{A}, \mathbb{B}}((T, t), \theta, \tau) \rightarrow \chi_{\mathbb{A}, \mathbb{B}}\left(\left(T^{\prime}, t^{\prime}\right), \theta^{\prime}, \tau^{\prime}\right)$. 
It consists of the horizontal 1-cell in $\mathbb{M n d}(\mathbb{B})$

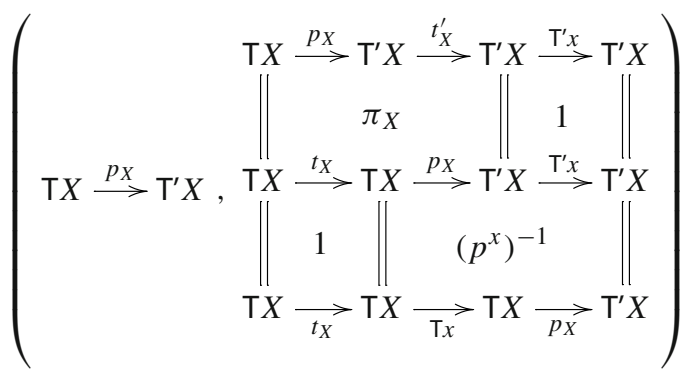

for all 0-cells $((X, x), \mu, \eta)$ in $\mathbb{M n d}(\mathbb{A})$ together with the 2-cell $p_{g}$ in $\mathbb{M}$ nd $(\mathbb{B})$ for all vertical 1 -cells $g$ in $\mathbb{M n d}(\mathbb{A})$, and the vertically invertible 2-cell $p^{h}$ in $\mathbb{M} n d(\mathbb{B})$ for all horizontal 1cells $h$ in $\mathbb{M n d}(\mathbb{A})$.

A vertical 1-cell

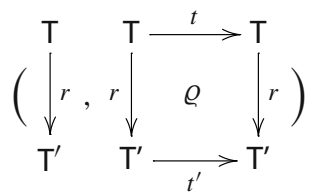

of $\mathbb{M n d} \llbracket \mathbb{A}, \mathbb{B} \rrbracket$ is sent by $\chi_{\mathbb{A}, \mathbb{B}}$ to the vertical pseudo transformation from $\chi_{\mathbb{A}, \mathbb{B}}((T, t), \theta, \tau)$ to $\chi_{\mathbb{A}, \mathbb{B}}\left(\left(T^{\prime}, t^{\prime}\right), \theta^{\prime}, \tau^{\prime}\right)$ which consists of the following data.

For all 0-cells $((X, x), \mu, \eta)$ of $\mathbb{M} n d(\mathbb{A})$ the vertical 1-cell in $\mathbb{M n d}(\mathbb{B})$

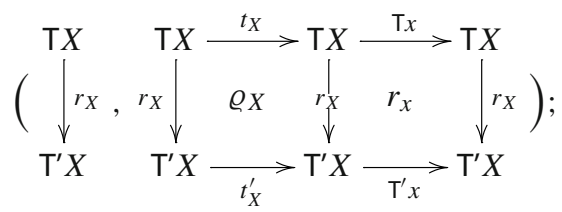

for all horizontal 1-cells $h$ of $\mathbb{M n d}(\mathbb{A})$ the 2-cell $r_{h}$ in $\mathbb{M n d}(\mathbb{B})$; and for all vertical 1-cells $g$ of $\mathbb{M n d}(\mathbb{A})$ the horizontally invertible 2-cell $r^{g}$ in $\mathbb{M n d}(\mathbb{B})$.

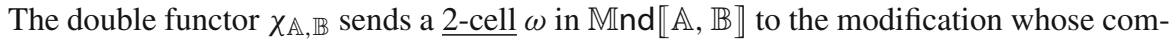
ponent at every 0 -cell $((X, x), \mu, \eta)$ of $\mathbb{M n d}(\mathbb{A})$ is $\omega_{X}$.

These double functors $\chi_{\mathbb{A}, \mathbb{B}}: \mathbb{M n d} \llbracket \mathbb{A}, \mathbb{B} \rrbracket \rightarrow \llbracket \mathbb{M n d}(\mathbb{A}), \mathbb{M n d}(\mathbb{B}) \rrbracket$ constitute a natural transformation $\chi: \mathbb{M n d} \llbracket-,-\rrbracket \rightarrow \llbracket \mathbb{M n d}(-), \mathbb{M} n d(-) \rrbracket$. It is not hard (although a bit long) to see that the double functors of Sect. 4.2, the trivial isomorphism $\mathbb{1} \cong \mathbb{M n d}(\mathbb{1})$ and the double functors $\chi_{\mathbb{A}, \mathbb{B}}$ constructed above, satisfy the conditions of (4.2) and (4.3). This proves the monoidality of the functor in the title of the section (which sends then monoids as in Sect. 4.3 to monoids in the same sense).

\subsection{The Closed Monoidal Category (DbICat, x)}

Recall that for any double category $\mathbb{A}$, the internal hom functor $\langle\mathbb{A},-\rangle:$ DblCat $\rightarrow$ DblCat of the closed monoidal category in the title sends an object; that is, a double category $\mathbb{B}$ to the following double subcategory $\langle\mathbb{A}, \mathbb{B}\rangle$ of $\llbracket \mathbb{A}, \mathbb{B} \rrbracket$.

- The $\underline{0 \text {-cells }}$ are still the double functors $\mathbb{A} \rightarrow \mathbb{B}$. 
- The horizontal 1-cells are the horizontal transformations of [15]. That is, those horizontal pseudo transformations $x$ (see Sect. 2.2) whose 2-cell parts $x^{h}$ are vertical identity 2-cells for all horizontal 1-cells $h$ in $\mathbb{A}$.

- Symmetrically, the vertical 1-cells are the vertical transformations of [15]. That is, those vertical pseudo transformations $y$ (see Sect. 2.2) whose 2-cell parts $y^{f}$ are horizontal identity 2-cells for all vertical 1-cells $f$ in $\mathbb{A}$.

- Finally, the 2-cells are the modifications of [15] (this is the same notion as in Sect. 2.2).

The functor $\langle\mathbb{A},-\rangle:$ DblCat $\rightarrow$ DblCat sends a morphism; that is, a double functor $\mathrm{H}: \mathbb{B} \rightarrow$ $\mathbb{C}$ to the restriction $\langle\mathbb{A}, H\rangle:\langle\mathbb{A}, \mathbb{B}\rangle \rightarrow\langle\mathbb{A}, \mathbb{C}\rangle$ of the double functor $\llbracket \mathbb{A}, H \rrbracket: \llbracket \mathbb{A}, \mathbb{B} \rrbracket \rightarrow$ $\llbracket \mathbb{A}, \mathbb{C} \rrbracket$ in Sect. 2.3.

For any double category $\mathbb{A}$, the double functor

$$
\mathbb{1} \stackrel{\eta_{\times}^{\mathbb{A}}}{\longrightarrow}\langle\mathbb{A}, \mathbb{1} \times \mathbb{A}\rangle \stackrel{\left\langle 1, \lambda_{\times}\right\rangle}{\longrightarrow}\langle\mathbb{A}, \mathbb{A}\rangle
$$

is $1_{\mathbb{A}}$, sending the single object of $\mathbb{1}$ to the identity double functor $1_{\mathbb{A}}: \mathbb{A} \rightarrow \mathbb{A}$; and the double functor

$$
\mathbb{A} \stackrel{\eta_{\times}^{\mathbb{1}}}{\longrightarrow}\langle\mathbb{1}, \mathbb{A} \times \mathbb{1}\rangle \stackrel{\left\langle 1, \varrho_{\times}\right\rangle}{\longrightarrow}\langle\mathbb{1}, \mathbb{A}\rangle
$$

is the canonical isomorphism $\mathbb{A} \cong\langle\mathbb{1}, \mathbb{A}\rangle \cong \llbracket \mathbb{1}, \mathbb{A} \rrbracket$.

For any double categories $\mathbb{A}, \mathbb{B}$ and $\mathbb{C}$, the double functor $l_{\times}^{\mathbb{C}}:\langle\mathbb{A}, \mathbb{B}\rangle \rightarrow\langle\langle\mathbb{C}, \mathbb{A}\rangle,\langle\mathbb{C}, \mathbb{B}\rangle\rangle$ of (4.1) is constituted by the following maps.

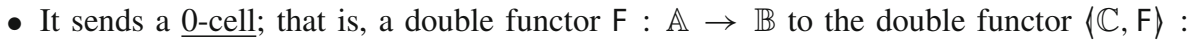
$\langle\mathbb{C}, \mathbb{A}\rangle \rightarrow\langle\mathbb{C}, \mathbb{B}\rangle$.

- It sends a horizontal 1-cell; that is, a horizontal transformation $\mathrm{F} \stackrel{x}{\rightarrow} \mathrm{G}$ to the horizontal transformation $\langle\mathbb{C}, F\rangle \rightarrow\langle\mathbb{C}, G\rangle$ whose component at any vertical transformation on the left-between double functors $\mathbb{C} \rightarrow \mathbb{A}$-is the modification on the right:

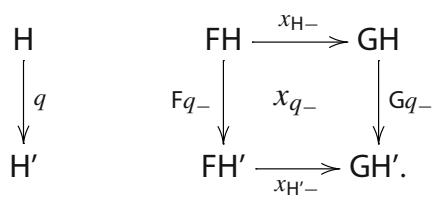

- Symmetrically, it sends a vertical 1-cell; that is, a vertical transformation on the leftbetween double functors $\mathbb{A} \rightarrow \mathbb{B}$-to the vertical transformation whose component at any horizontal transformation $\mathrm{H} \stackrel{p}{\rightarrow} \mathrm{H}^{\prime}$ - between double functors $\mathbb{C} \rightarrow \mathbb{A}$-is the modification on the right:

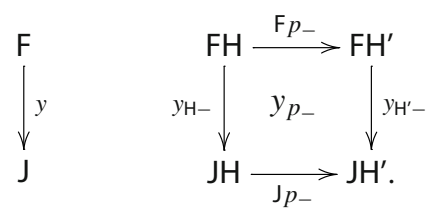

- Finally, it sends a modification on the left to the modification whose component at a double functor $\mathrm{H}: \mathbb{C} \rightarrow \mathbb{A}$ is the modification on the right: 

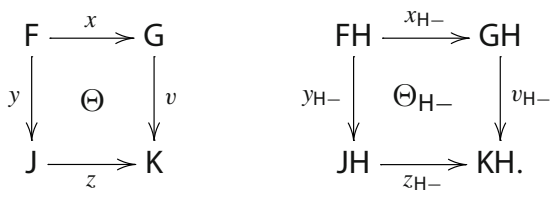

\subsection{Monoidality of the Identity Functor (DbICat, $x) \rightarrow($ DblCat, $\otimes)$}

The evident inclusion double functors $\langle\mathbb{A}, \mathbb{B}\rangle \succ \llbracket \mathbb{A}, \mathbb{B} \rrbracket$, for all double categories $\mathbb{A}$ and $\mathbb{B}$, define a natural transformation $\langle-,-\rangle \longmapsto \llbracket-,-\rrbracket$. Together with the double functors in Sect. 4.2 and those in Sect. 4.5, and the identity double functor $\mathbb{1}=\mathbb{1}$ as the nullary part of the stated monoidal structure, they clearly satisfy the conditions in (4.2) and (4.3). With this we infer the monoidality of the functor in the title of the section.

In particular, a strict monoidal double category [7] — which is a monoid in (DblCat, $\times$ ) gives rise to a monoid in (DblCat, $\otimes)$-described in Sect. 4.3.

\subsection{The Closed Monoidal Category (2-Cat, $\otimes)$}

In this section we regard the category 2-Cat of 2-categories and 2-functors as a closed monoidal category via the Gray monoidal product $\otimes$ in [19]. Recall that, for any 2-category $\mathcal{A}$, the internal hom functor $[\mathcal{A},-]: 2$-Cat $\rightarrow$ 2-Cat sends an object; that is, a 2-category $\mathcal{B}$ to the 2-category $[\mathcal{A}, \mathcal{B}]$ of 2-functors $\mathcal{A} \rightarrow \mathcal{B}$, pseudo natural transformations, and modifications. It sends a morphism; that is, a 2-functor $\mathrm{F}: \mathcal{B} \rightarrow \mathcal{C}$ to the 2-functor $[\mathcal{A}, \mathrm{F}]:[\mathcal{A}, \mathcal{B}] \rightarrow[\mathcal{A}, \mathcal{C}]$ given by postcomposition with $\mathrm{F}$.

The 2-functor

$$
1 \stackrel{\eta^{\mathcal{A}}}{\longrightarrow}[\mathcal{A}, 1 \otimes \mathcal{A}] \stackrel{[1, \lambda]}{\longrightarrow}[\mathcal{A}, \mathcal{A}]
$$

is $1_{\mathcal{A}}$, the 2 -functor sending the single object of the terminal 2-category 1 to the identity 2-functor $1_{\mathcal{A}}: \mathcal{A} \rightarrow \mathcal{A}$.

The 2-functor

$$
\mathcal{A} \stackrel{\eta^{l}}{\longrightarrow}[1, \mathcal{A} \otimes 1] \stackrel{[1, \varrho]}{\longrightarrow}[1, \mathcal{A}]
$$

is the canonical isomorphism.

For any 2-categories $\mathcal{A}, \mathcal{B}$ and $\mathcal{C}$, the 2-functor $\mathfrak{l}_{\mathcal{A}, \mathcal{B}}:[\mathcal{A}, \mathcal{B}] \rightarrow[[\mathcal{C}, \mathcal{A}],[\mathcal{C}, \mathcal{B}]]$ in $(4.1)$ has the following maps.

- It sends a 0 -cell; that is, a 2-functor $\mathrm{H}: \mathcal{A} \rightarrow \mathcal{B}$ to the 2-functor $[\mathcal{C}, \mathrm{H}]:[\mathcal{C}, \mathcal{A}] \rightarrow[\mathcal{C}, \mathcal{B}]$.

- It sends a $\underline{1-c e l l}$; that is, a pseudo natural transformation $\psi: \mathrm{H} \rightarrow \mathrm{H}^{\prime}$ to the pseudo natural transformation $[\mathcal{C}, \mathrm{H}] \rightarrow\left[\mathcal{C}, \mathrm{H}^{\prime}\right]$ whose component at any pseudo natural transformation $\varphi: \mathrm{F} \rightarrow \mathrm{F}^{\prime}$ between 2-functors $\mathcal{C} \rightarrow \mathcal{A}$ is

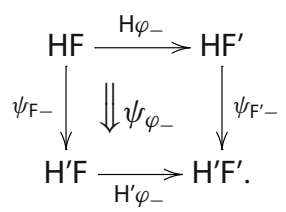

- It sends a 2-cell; that is, a modification $\omega$ to the modification whose component at any 2-functor $\mathrm{F}: \mathcal{C} \rightarrow \mathcal{A}$ is $\omega_{\mathrm{F}-}$. 


\subsection{Monoidality of the Functors $($ DblCat, $\otimes) \rightarrow(2-C a t, \otimes)$ Sending Double Categories to Their Horizontal-or Vertical-2-Categories}

Consider the functor $\mathcal{H}:$ DblCat $\rightarrow$ 2-Cat which sends a double category $\mathbb{A}$ to its so-called horizontal 2-category. The 0 -cells of $\mathcal{H} \mathbb{A}$ are the 0 -cells of $\mathbb{A}$, the 1-cells of $\mathcal{H} \mathbb{A}$ are the horizontal 1-cells of $\mathbb{A}$ and the 2-cells of $\mathcal{H} \mathbb{A}$ are those 2-cells of $\mathbb{A}$ which are surrounded by identity vertical 1-cells (and arbitrary horizontal 1-cells). Compositions in $\mathcal{H} \mathbb{A}$ are inherited from $\mathbb{A}$. The functor $\mathcal{H}$ sends a morphism; that is, a double functor $\mathrm{F}: \mathbb{A} \rightarrow \mathbb{B}$ to the 2 -functor $\mathcal{H} \mathrm{F}: \mathcal{H} \mathbb{A} \rightarrow \mathcal{H B}$ which acts on the various cells as $\mathrm{F}$ does.

The horizontal 2-category of the terminal double category $\mathbb{1}$ is the terminal 2-category 1 . So we may choose the nullary part $\mathcal{H}_{0}$ of the candidate monoidal structure on $\mathcal{H}$ to be the identity 2 -functor $1 \rightarrow 1$. As the 2 -functor $\chi_{\mathbb{A}, \mathbb{B}}: \mathcal{H} \llbracket \mathbb{A}, \mathbb{B} \rrbracket \rightarrow[\mathcal{H} \mathbb{A}, \mathcal{H} \mathbb{B}]$ for any double categories $\mathbb{A}$ and $\mathbb{B}$, encoding the binary part, we propose the following.

- A 0 -cell; that is, a double functor $\mathrm{F}: \mathbb{A} \rightarrow \mathbb{B}$ is sent to the 2-functor $\mathcal{H} F: \mathcal{H} \mathbb{A} \rightarrow \mathcal{H B}$.

- A 1-cell; that is, a horizontal pseudo transformation $x: \mathrm{F} \rightarrow \mathrm{G}$ is sent to the pseudo natural transformation $\mathcal{H F} \rightarrow \mathcal{H G}$ whose component at any 1-cell of $\mathcal{H} \mathbb{A}$ - that is, horizontal 1-cell $h: A \rightarrow C$ of $\mathbb{A}$-is the 2-cell $x^{h}: x_{C}$. F $h \rightarrow \mathrm{G} h \cdot x_{A}$ of $\mathcal{H B}$.

- A 2-cell; that is, a modification of the form

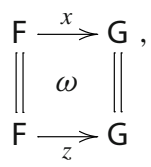

is sent to the modification whose component at any 0-cell of $\mathcal{H} \mathbb{A}$-that is, 0-cell $A$ of $\mathbb{A}$-is the 2-cell $\omega_{A}: x_{A} \rightarrow z_{A}$ of $\mathcal{H} \mathbb{B}$.

The so defined 2-functors $\chi_{\mathbb{A}, \mathbb{B}}$ constitute a natural transformation $\chi: \mathcal{H} \llbracket-,-\rrbracket \rightarrow$ $[\mathcal{H}-, \mathcal{H}-]$ and satisfy the conditions in (4.2) and (4.3). Hence there is a corresponding monoidal structure on $\mathcal{H}$. In particular, applying $\mathcal{H}$ to a monoid in (DblCat, $\otimes$ ) as in Sect. 4.3, we obtain a monoid in (2-Cat, $\otimes)$ (known as a Gray monoid [14]).

Symmetric considerations verify monoidality of the functor V : DblCat $\rightarrow 2$-Cat, sending a double category to its vertical 2-category.

\subsection{Monoidality of the Functor Sqr : $(2-C a t, \otimes) \rightarrow($ DblCat, $\otimes)$ Due to Ehresmann}

Ehresmann's square- or quintet construction [9] sends a 2-category $\mathcal{A}$ to the following double category $\operatorname{Sqr}(\mathcal{A})$. The 0 -cells of $\operatorname{Sqr}(\mathcal{A})$ are the 0 -cells of $\mathcal{A}$. Both the horizontal and the vertical 1-cells of $\operatorname{Sqr}(\mathcal{A})$ are the 1-cells of $\mathcal{A}$. A 2-cell of $\operatorname{Sqr}(\mathcal{A})$ with boundaries

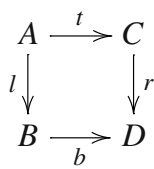

is a 2 -cell $r . t \rightarrow b . l$ of $\mathcal{A}$. For any 2 -functor $\mathrm{F}: \mathcal{A} \rightarrow \mathcal{B}$ there is a double functor $\operatorname{Sqr}(\mathrm{F})$ : $\operatorname{Sqr}(\mathcal{A}) \rightarrow \operatorname{Sqr}(\mathcal{B})$ which acts on the various cells as $\mathrm{F}$ does.

Applying the so defined functor $\mathbb{S}$ qr to the terminal 2-category 1, we obtain the terminal double category $\mathbb{1}$. So as the nullary part of the candidate monoidal structure on $\mathbb{S}$ qr, we may choose the identity double functor $\mathbb{1} \rightarrow \mathbb{1}$. For any 2-categories $\mathcal{A}$ and $\mathcal{B}$, for the double 
functor $\operatorname{Sqr}[\mathcal{A}, \mathcal{B}] \rightarrow \llbracket \operatorname{Sqr}(\mathcal{A}), \operatorname{Sqr}(\mathcal{B}) \rrbracket$ encoding the binary part, the following choices can be made.

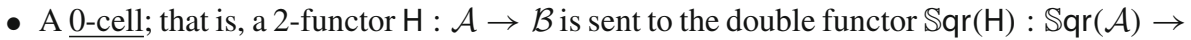
$\operatorname{Sqr}(\mathcal{B})$.

- A horizontal 1-cell; that is, a pseudo natural transformation $p: \mathrm{H} \rightarrow \mathrm{K}$ is sent to the horizontal pseudo transformation whose components at any 1-cell $f: X \rightarrow Y$ of $\mathcal{A}$ are the 2-cells in $\operatorname{Sqr}(\mathcal{B})$
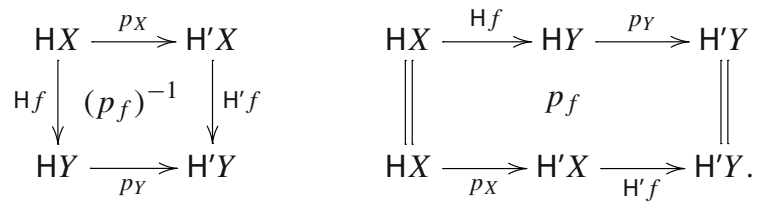

- A vertical 1-cell; which is again a pseudo natural transformation $p: \mathrm{H} \rightarrow \mathrm{K}$ is sent to the vertical pseudo transformation whose components at any 1-cell $f: X \rightarrow Y$ of $\mathcal{A}$ are the 2-cells in $\operatorname{Sqr}(\mathcal{B})$
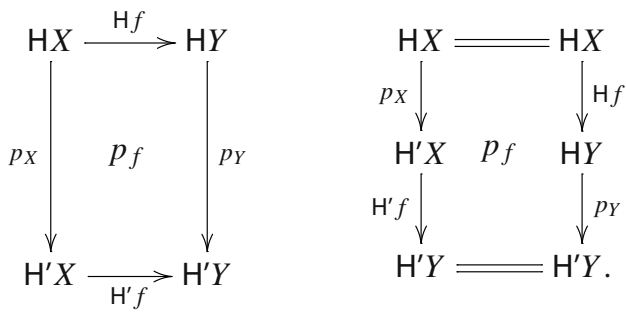

- A 2-cell; that is, a modification of 2-functors on the left, is sent to the modification of double functors whose component at any 0 -cell of $\operatorname{Sqr}(\mathcal{A})$ - that is, any 0-cell $X$ of $\mathcal{A}$-is the 2-cell of $\operatorname{Sqr}(\mathcal{B})$ on the right:
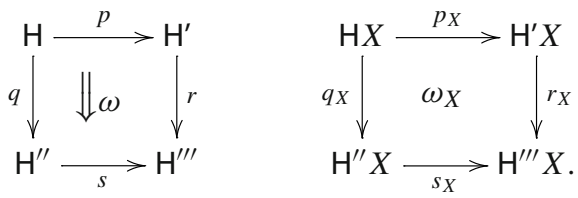

The resulting double functors $\operatorname{Sqr}[\mathcal{A}, \mathcal{B}] \rightarrow \llbracket \operatorname{Sqr}(\mathcal{A}), \operatorname{Sqr}(\mathcal{B}) \rrbracket$ constitute a natural transformation $\operatorname{Sqr}[-,-] \rightarrow \llbracket \mathbb{S q r}(-), \operatorname{Sqr}(-) \rrbracket$ and satisfy the conditions in (4.2) and (4.3). So they render monoidal the functor in the title of the section. In particular, applying the functor $\mathbb{S q r}$ to a Gray monoid - that is, a monoid in $(2-C a t, \otimes)$ - a monoid in (DblCat, $\otimes$ ) is obtained (which may not be a monoidal bicategory in the sense of [24, Definition 2.9]).

Acknowledgements Open access funding provided by MTA Wigner Research Centre for Physics (MTA Wigner FK, MTA EK). Financial support by the Hungarian National Research, Development and Innovation Office-NKFIH (Grant K124138) is gratefully acknowledged.

Open Access This article is distributed under the terms of the Creative Commons Attribution 4.0 International License (http://creativecommons.org/licenses/by/4.0/), which permits unrestricted use, distribution, and reproduction in any medium, provided you give appropriate credit to the original author(s) and the source, provide a link to the Creative Commons license, and indicate if changes were made. 


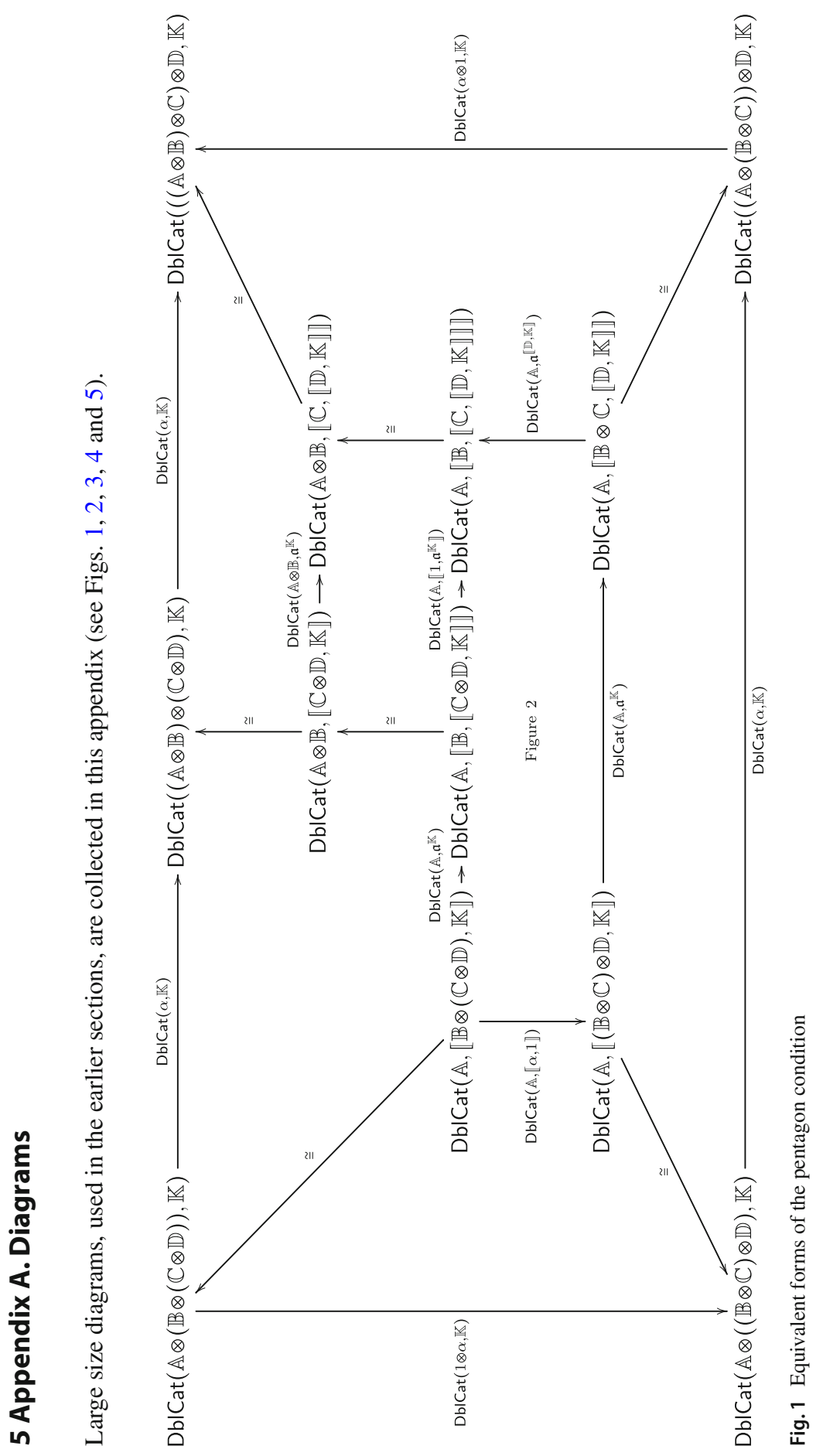




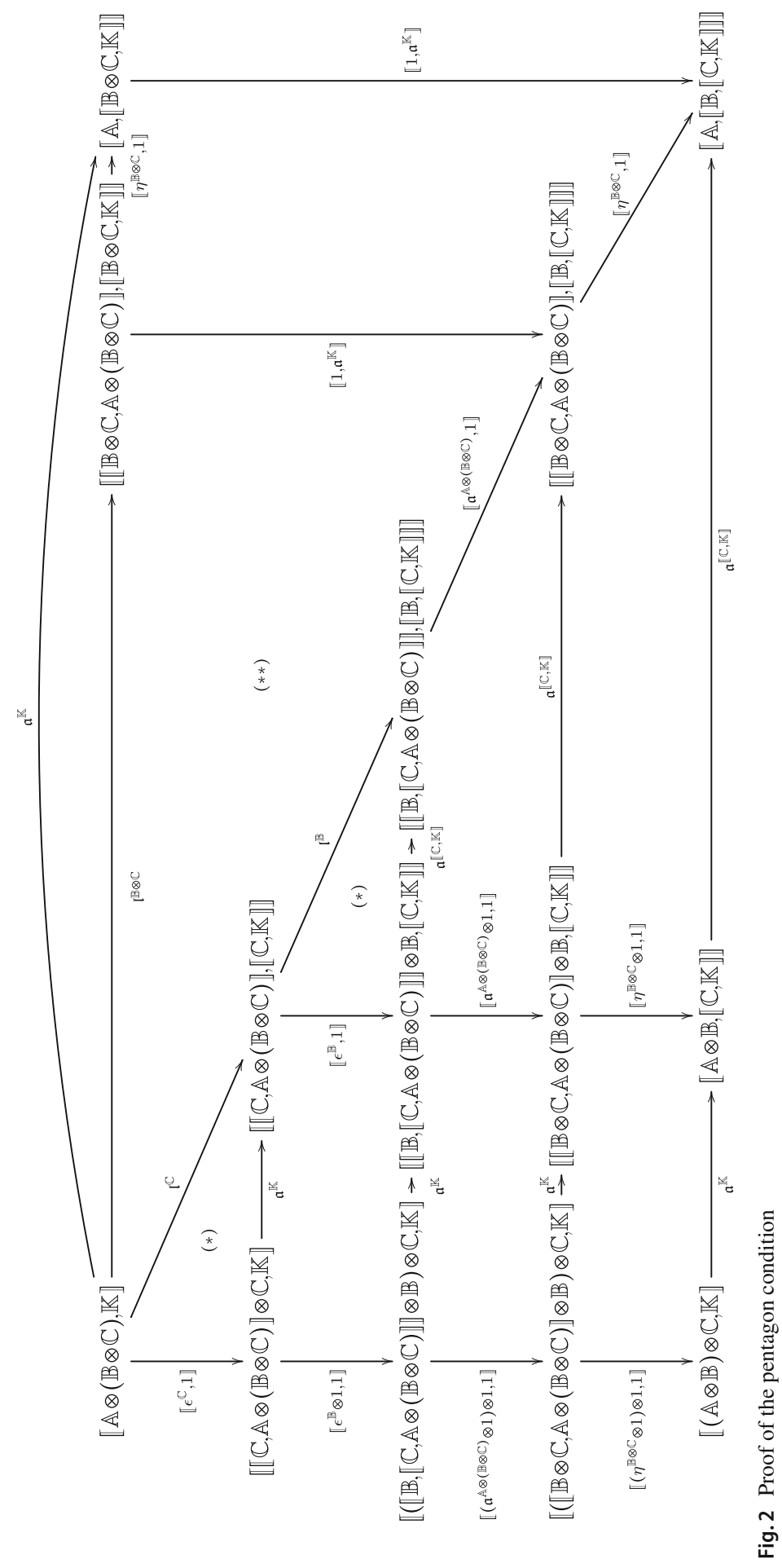




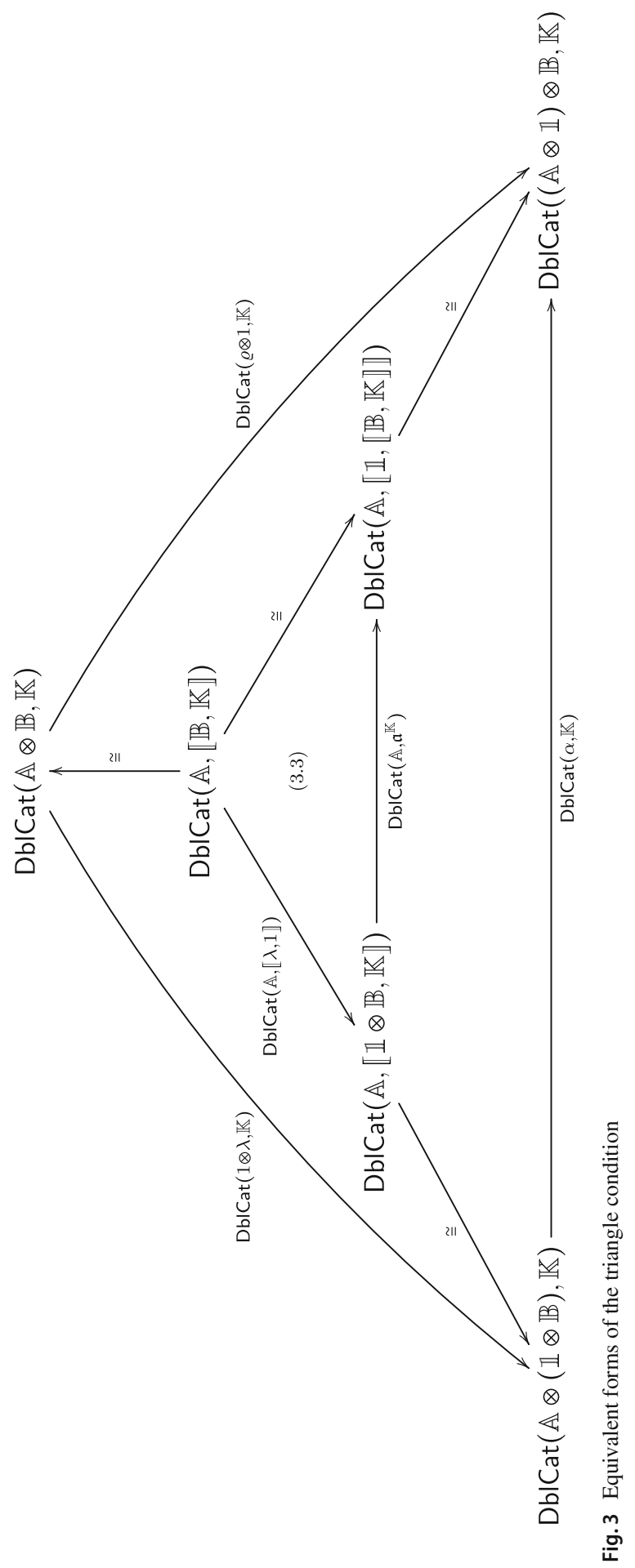




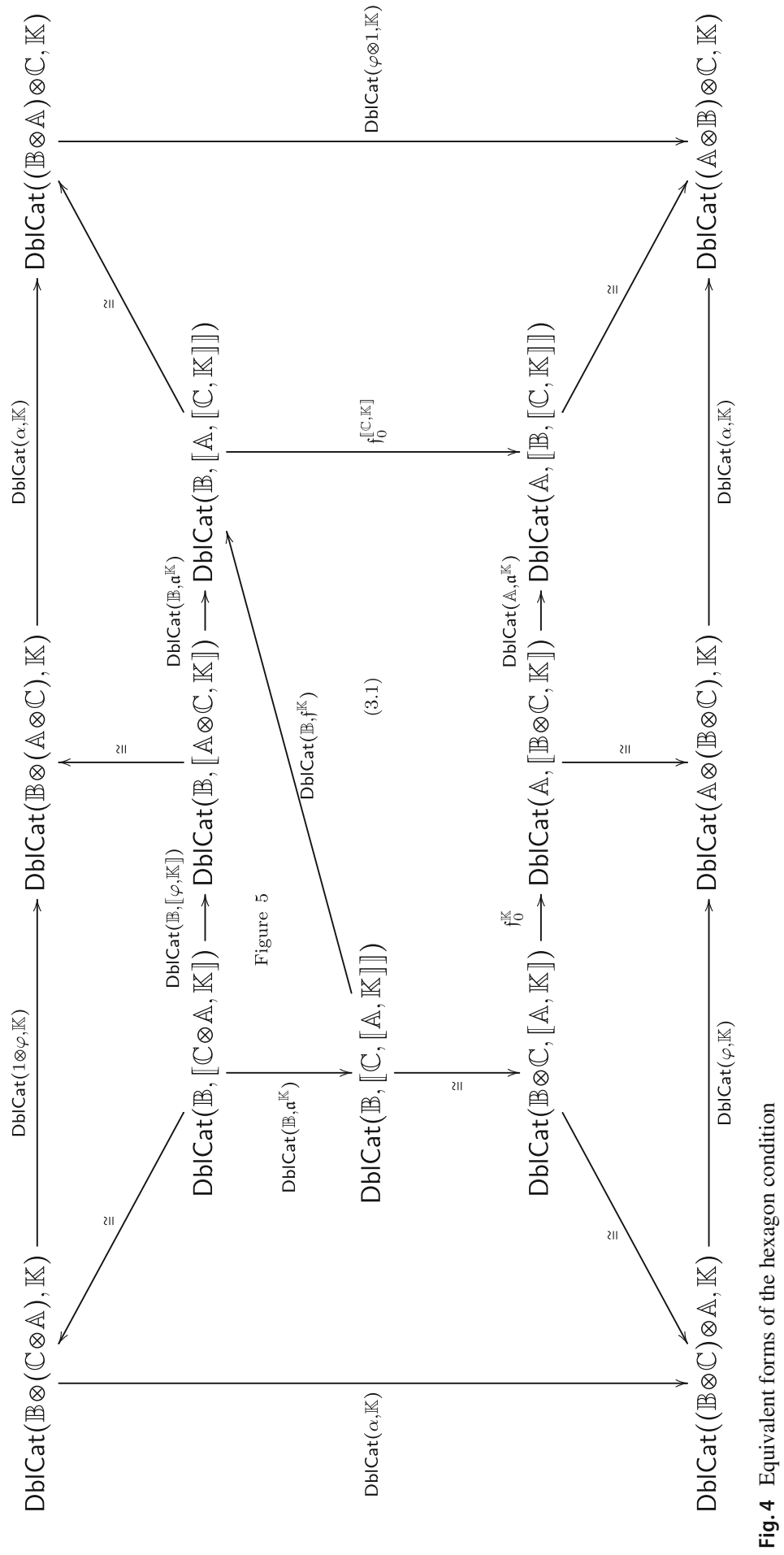




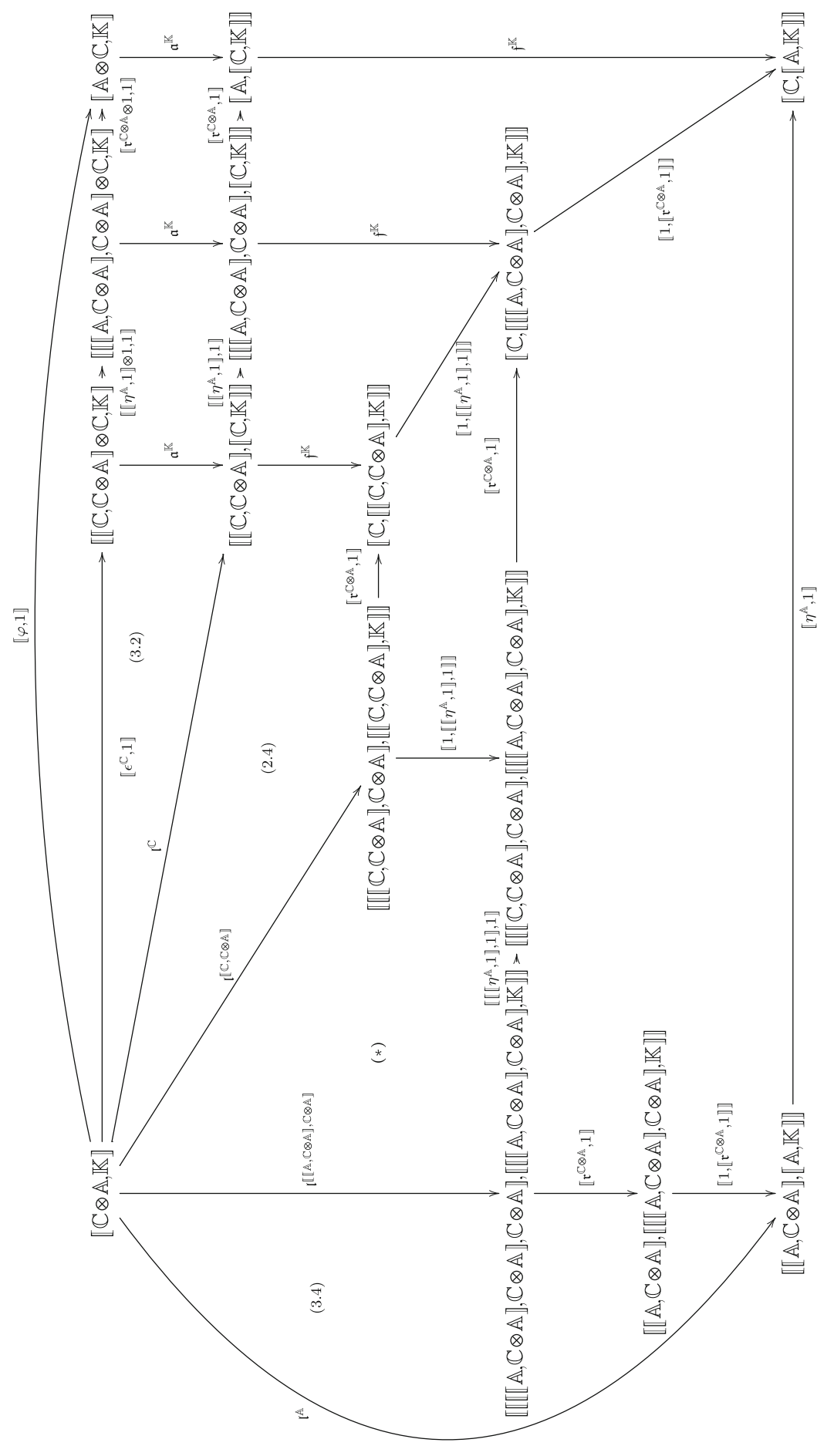

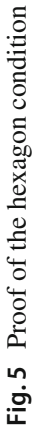




\section{References}

1. Aguiar, M., Mahajan, S.: Monoidal Functors, Species and Hopf Algebras. CRM Monograph Series 29. American Mathematical Society, Providence (2010)

2. Aguiar, M., Haim, M., López Franco, I.: Monads on higher monoidal categories. Appl. Categ. Struct. 26(3), 413-458 (2018)

3. Baez, J.C., Neuchl, M.: Higher-dimensional algebra. I. Braided monoidal 2-categories. Adv. Math. 121(2), 196-244 (1996)

4. Böhm, G.: The formal theory of multimonoidal monads. Theory Appl. Categ. 34(12), 295-348 (2019)

5. Borceux, F.: Handbook of Categorical Algebra: Basic Category Theory, vol. 1. Cambridge University Press, Cambridge (1994)

6. Bourke, J., Gurski, N.: The Gray tensor product via factorisation. Appl. Categ. Struct. 25(4), 603-624 (2017)

7. Bruni, R., Meseguer, J., Montanari, U.: Symmetric monoidal and cartesian double categories as a semantic framework for tile logic. Math. Struct. Comput. Sci. 12(1), 53-90 (2002)

8. Day, B.J., Laplaza, M.L.: On embedding closed categories. Bull. Aust. Math. Soc. 18(3), 357-371 (1978)

9. Ehresmann, C.: Catégories structurées III. Quintettes et applications covariantes. Séminaire Ehresmann. Topologie et géométrie différentielle 5, 1-21 (1963)

10. Fiore, T.M., Gambino, N., Kock, J.: Monads in double categories. J. Pure Appl. Algebra 215(6), 11741197 (2011)

11. Fiore, T.M., Paoli, S., Pronk, D.: Model structures on the category of small double categories. Algebr. Geom. Topol. 8, 1855-1959 (2008)

12. Garner, R.: Double clubs. Cahiers de Topologie et Géométrie Différentielle Catégoriques 47(4), 261-317 (2006)

13. Garner, R., Gurski, N.: The low-dimensional structures formed by tricategories. Math. Proc. Camb. Philos. Soc. 146(3), 551-589 (2009)

14. Gordon, R., Power, A.J., Street, R.: Coherence for tricategories. Memoirs of the American Mathematical Society 117(558), (1995)

15. Grandis, M., Paré, R.: Limits in double categories. Cahiers de Topologie et Géométrie Différentielle Catégoriques 40(3), 162-220 (1999)

16. Grandis, M., Paré, R.: Adjoint for double categories. Cahiers de Topologie et Géométrie Différentielle Catégoriques 45(3), 193-240 (2004)

17. Grandis, M., Paré, R.: Intercategories. Theory Appl. Categ. 30(38), 1215-1255 (2015)

18. Grandis, M., Paré, R.: Intercategories: a framework for three-dimensional category theory. J. Pure Appl. Algebra 221(5), 999-1054 (2017)

19. Gray, J.W.: Formal Category Theory: Adjointness for 2-Categories. Lecture Notes in Mathematics, vol. 391. Springer, Berlin (1974)

20. Kapranov, M.M., Voevodsky, V.: 2-categories and Zamolodchikov tetrahedra equations. In: Haboush, W.J., Parshall, B.J. (eds.) Algebraic Groups and Their Generalizations: Quantum and Infinite-Dimensional Methods. Proceedings of Symposia in Pure Mathematics, vol. 56, pp. 177-259. American Mathematical Society, Providence, RI (1994)

21. Mac Lane, S.: Categories for the Working Mathematician. Graduate Texts in Mathematics, vol. 5, 2nd edn. Springer, Berlin (1978)

22. Schommer-Pries, C.: The Classification of Two-Dimensional Extended Topological Field Theories. Ph.D. Dissertation, University of California, Berkeley, (2009). arXiv:1112.1000

23. Shulman, M.A.: Comparing composites of left and right derived functors. N. Y. J. Math. 17, 75-125 (2011)

24. Shulman, M.A.: Constructing symmetric monoidal bicategories, arXiv:1004.0993

Publisher's Note Springer Nature remains neutral with regard to jurisdictional claims in published maps and institutional affiliations. 